\title{
Mechanisms for the mitigation of the hose instability in plasma-wakefield accelerators
}

\author{
T. J. Mehrling, ${ }^{1,2,3,4, *}$ R. A. Fonseca, ${ }^{4,5}$ A. Martinez de la Ossa, ${ }^{3}$ and J. Vieira ${ }^{4, \dagger}$ \\ ${ }^{1}$ Lawrence Berkeley National Laboratory, Berkeley, California 94720, USA \\ ${ }^{2}$ Deutsches Elektronen-Synchrotron DESY, Notkestrasse 85, 22607 Hamburg, Germany \\ ${ }^{3}$ Institut für Experimentalphysik, Universität Hamburg, 22761 Hamburg, Germany \\ ${ }^{4}$ GoLP/Instituto de Plasmas e Fusão Nuclear, Instituto Superior Técnico, Universidade de Lisboa, \\ 1049-001 Lisboa, Portugal \\ ${ }^{5}$ DCTI/ISCTE Instituto Universitário de Lisboa, 1649-026 Lisbon, Portugal
}

(Received 21 February 2019; published 19 March 2019)

\begin{abstract}
In this work we provide an in-depth analysis of mechanisms which mitigate the hose instability in the blowout regime in plasma-wakefield accelerators. We show by means of theory and three-dimensional particle-in-cell simulations that the mitigation mechanisms related to a beam energy spread are effective for parameters as used in major plasma-wakefield accelerator experiments and for various beam energies, beam current profiles, and types of hosing seed. In addition, we establish the theoretical principles for the reduction of the initial hosing seed in tapered vacuum-to-plasma transitions and derive respective analytic predictions which are successfully benchmarked against particle-in-cell simulations. We also investigate the possibility to facilitate efficient and stable acceleration of witness beams in the blowout regime. This work therefore provides a deepened understanding for the methods that allow for the mitigation of hosing, a crucial prerequisite to facilitate stable acceleration of high quality beams in plasma-wakefield accelerators.
\end{abstract}

DOI: 10.1103/PhysRevAccelBeams.22.031302

\section{INTRODUCTION}

Plasma-based accelerators can potentially leverage a dramatic miniaturization of future accelerators and provide improvements in terms of provided beam energy, versatility, and availability of accelerator facilities. In plasma-wakefield accelerators (PWFAs) [1,2], short and high-current particle beams excite plasma waves which carry extreme accelerating fields in excess of $10 \mathrm{GV} / \mathrm{m}$ [3]. Drive beams with a charge density much greater than the ambient plasma density expel all plasma electrons near the propagation axis, such that an ion cavity (plasma channel) is formed which copropagates behind the drive beam. This excitation regime, the blowout regime, features an enhanced accelerating field and transverse linearly focusing forces for trailing relativistic electron beams [4].

Trailing electron beams can be injected into the plasma wave externally [5], through controlled wave-breaking from the plasma [6] or via ionization from a dopand gas [7-9]. These techniques promise the generation of beams

\footnotetext{
*timon.mehrling@desy.de

jorge.vieira@ist.utl.pt
}

Published by the American Physical Society under the terms of the Creative Commons Attribution 4.0 International license. Further distribution of this work must maintain attribution to the author(s) and the published article's title, journal citation, and DOI. with a sufficient quality to drive pivotal applications such as free-electron lasers. However, in addition to the demand of high beam quality, these applications require a high stability of the beam parameters.

In this context, the hose instability (or beam-breakup instability) is since long predicted to be a major impediment for the applicability of PWFAs [10]. The hose instability, which is seeded by initial transverse beam and/or plasma phase space asymmetries, is growing rapidly due to the extreme transverse fields, which can be on the same order of magnitude as the longitudinal fields in the blowout regime. According to current models, the beam centroid displacement is amplified exponentially during the beam propagation in the plasma, resulting in an unstable acceleration process or in beam-breakup [10-14].

It was recently shown that these models overestimate the hosing growth rates as soon as the intrinsic drive-beam energy change becomes significant [15], suggesting that the blowout regime in PWFAs can provide intrinsic saturation mechanisms for the hose instability. These effects are similar to Balakin-Novokhatsky-Smirnov damping [16] in conventional accelerators and similar to the mitigation of hosing through a change of the betatron frequency for self-modulated long beams in PWFAs [17], beams in the linear regime [18] or wide drive beams in PWFAs [19]. The mechanisms associated to an energy spread of drive beams, leading to the mitigation of hosing in the blowout regime in PWFAs were outlined in Ref. [15] and their capability to 
substantially mitigate the beam centroid oscillations during propagation in the plasma was shown. These results indicated for the first time the possibility of stable acceleration of beams over long distances in PWFAs.

In Sec. II of this work, we review the state of the art description of the hose instability. We then present a detailed derivation of the new beam centroid equation in Sec. III. The implications of this equation are investigated in Sec. IV, and it is shown analytically that the intrinsic beam energy change and an energy spread provide effective mitigation mechanisms for a wide range of PWFA scenarios. In Sec. V, particle-in-cell (PIC) simulation results with the quasistatic code HIPACE [20] are presented which demonstrate the mitigation of hosing for setups similar to those used in existing facilities or as anticipated for future facilities and which provide new insights into the underlying physics. Additionally, in Sec. VI, we explicate how tapered vacuum-to-plasma transitions reduce initial seeds for hosing and/or witness-drive misalignments. We provide successful comparisons between our model and simulation results using the PIC framework OSIRIS [21]. We also investigate the possibility to mitigate the hosing of the witness beam in a PWFA-driven collider scheme in Sec. VII. The summary and conclusion in Sec. VIII finalize this paper.

\section{REVIEW OF THE HOSE INSTABILITY}

The hose instability is seeded by transverse asymmetries of the beam or plasma phase space distributions. For example, an asymmetry of the transverse plasma density distribution leads to a deviation of the ion-channel, or plasma centroid $X_{p}$ along the beam. This results in a respective temporal evolution of the beam centroid deviation $X_{b}$, which in turn drives a displacement of the ion-channel centroid $X_{p}$ along the beam, and so on. The current mathematical description of this coupled evolution of the ion-channel centroid $X_{p}(\xi, t)$ and the beam centroid $X_{b}(\xi, t)$ is [22]

$$
\begin{gathered}
\frac{\partial^{2} X_{p}}{\partial \xi^{2}}+\mathcal{C}_{d}(\xi) \frac{\partial X_{p}}{\partial \xi}+\frac{\mathcal{C}_{p}(\xi)}{2} X_{p}=\frac{\mathcal{C}_{b}(\xi)}{2} X_{b} \\
\frac{\partial^{2} X_{b}}{\partial t^{2}}+\omega_{\beta}^{2} X_{b}=\omega_{\beta}^{2} X_{p}
\end{gathered}
$$

where $t$ is the time and $\xi=c t-z$ the comoving coordinate, and where $z$ is the propagation axis and $c$ the speed of light. The plasma wave number is denoted by $k_{p}=\omega_{p} / c$ and the betatron frequency by $\omega_{\beta}=\omega_{p} / \sqrt{2 \gamma}$, with the Lorentz factor $\gamma$ of beam electrons, and where $\omega_{p}=\sqrt{4 \pi n_{0} e^{2} / m}$ is the plasma frequency with the ambient plasma density $n_{0}$, the elementary charge $e$ and the electron rest mass $m$.

According to Eq. (1), the beam centroid displacement $X_{b}$ drives a plasma centroid displacement $X_{p}$ proportionally to
$\mathcal{C}_{b}(\xi)$. The coefficient $\mathcal{C}_{p}(\xi)$ accounts for the restoring force exerted by the wakefields onto the plasma sheath electrons to a perturbation $X_{p}$. Sheath electrons undergo dynamics which cause a relativistic mass gain or loss. The associated damping or amplifying effect is reproduced by the coefficient $\mathcal{C}_{d}(\xi)$ [22]. The case with $\mathcal{C}_{p}=\mathcal{C}_{b}=1$ and $\mathcal{C}_{d}=0$ recovers the original hosing model [10]. This limit assumes an adiabatically generated, nonrelativistic ion channel with a $\xi$-independent response of sheath electrons. In is limit, $X_{b}$ and $X_{p}$ grow exponentially along $\xi$ owing to hosing [10-12]. In addition, since the above models assume a monoenergetic beam with constant energy, the growth of $X_{b}$ and $X_{p}$ is exponential in time $[11,12,14]$.

The hose instability is a result of a coherent coupling of transverse phase space asymmetries of beam particles and plasma electrons. Hence, the hose instability is mitigated if the coupling between beam and plasma is reduced, if initial beam or plasma phase space asymmetries are reduced, and/or if the coherence of beam or plasma oscillations is disrupted. It was recently shown that the relativistic motion of electrons in the plasma electron sheath and the channel radius greater than the charge-neutralization radius in the non-adiabatic, relativistic ion-channel (i.e., blowout) regime can reduce the coupling, and thereby decrease the growth rate of the hose instability if $C_{b}<1$ [14,22]. While this indicates a reduction of the hosing growth rate in the blowout regime, the growth is predicted to be exponential in time. Hence, small asymmetries eventually lead to beam breakup during propagation in the plasma according to models which do not incorporate beam energy spread or energy change.

Here, we incorporate the beam energy loss into the model, which, in agreement with energy conservation, intrinsically occurs as the drive beam excites the plasma wave, and show how it results in a decoherence of the betatron oscillation phases along the beam. It is also shown that an initial correlated energy spread leads to a decoherence of the betatron oscillations between various slices. Both latter effects imply a saturation of the hose instability. In addition, we explicate how a finite uncorrelated energy spread results in a decoherence of the electron oscillations within individual slices and thereby damps hosing. Finally, a technique for the reduction of initial beam asymmetries which seed hosing is proposed and described.

\section{DERIVATION OF THE BEAM-CENTROID EQUATION WITH ENERGY EFFECTS}

\section{A. Dynamics of single beam electrons}

We generalize the beam centroid equation (2) in order to establish a model that incorporates the effects of beam energy spread and energy change. We do so by starting from the single-electron motion which is then averaged over the initial beam phase space distribution. 
The transverse position $x$ of a single beam electron relative to the axis in a homogeneous ion-channel is described by the differential equation $[23,24]$

$$
\frac{d^{2} x}{d t^{2}}+\frac{\dot{\gamma}}{\gamma} \frac{d x}{d t}+\omega_{\beta}^{2} x=\omega_{\beta}^{2} X_{p},
$$

where the dot above the Lorentz factor $\gamma$ refers to the time derivative, i.e., $\dot{\gamma}=d \gamma / d t$. The beam electrons have a great longitudinal momentum $p_{z} \gg m c$, such that $\gamma \simeq$ $p_{z} / m c \gg 1$ is decoupled from the transverse motion, since the transverse motion is nonrelativistic $d x / d t \ll c$. The restoring force is directed toward the instantaneous position of the channel centroid $X_{p}$. Radiation effects are neglected, which, otherwise, can result in a growth of the energy spread of the beam [25]. According to Eq. (3), the oscillation amplitude of an electron which gains energy $(\dot{\gamma}>0)$ is damped while the amplitude of an electron losing energy $(\dot{\gamma}<0)$ is amplified.

The electron energy change occurs on a timescale much greater than the oscillation period. Taking this fact into account, one finds for the solution of Eq. (3)

$$
\begin{aligned}
x(t) \simeq & x_{0} A(t) \cos [\varphi(t)]+\frac{p_{x, 0}}{m \gamma_{0} \omega_{\beta, 0}} A(t) \sin [\varphi(t)] \\
& +\omega_{\beta, 0} \int_{0}^{t} A(t) A\left(t^{\prime}\right) \sin \left[\varphi(t)-\varphi\left(t^{\prime}\right)\right] X_{p}\left(t^{\prime}\right) \mathrm{d} t^{\prime} .
\end{aligned}
$$

Here, $\omega_{\beta, 0}=\omega_{p} / \sqrt{2 \gamma_{0}}$ denotes the initial betatron frequency and $A(t)=\left[\gamma_{0} / \gamma(t)\right]^{1 / 4}$ the oscillation amplitude, and where $\gamma_{0}=\gamma(t=0)$ and $p_{x, 0}=p_{x}(t=0)$ refer to the initial Lorentz factor and transverse momentum, respectively. The individual phase-advance of electrons is $\varphi(t)=$ $\int \omega_{\beta} \mathrm{d} t$. We neglected the terms $\mathcal{O}\left[\dot{\gamma} \dot{A} /\left(\dot{\varphi}^{2} \gamma A\right)\right] \ll 1$, $\mathcal{O}\left[\ddot{A} /\left(\dot{\varphi}^{2} A\right)\right] \ll 1$, and $\mathcal{O}\left[\dot{\gamma} /\left(4 \gamma_{0} \omega_{\beta, 0}\right)\right] \ll 1$ which are small since the relative energy and amplitude variations occur on timescales longer than the betatron period.

The energy of a beam electron may have an initial deviation $\delta \gamma$ with respect to the initial mean energy of a beam slice $\bar{\gamma}_{0}$ and be temporally evolving with an acceleration rate $\mathcal{E}$. Hence, the electron energy is represented by $\gamma(t)=\bar{\gamma}_{0}+\mathcal{E} t+\delta \gamma$. Note that the beam may have an initial energy chirp and hence, the initial mean slice energy, generally, is a function of the comoving coordinate $\bar{\gamma}_{0}=\bar{\gamma}_{0}(\xi)$. The same is true for the rate of energy change $\mathcal{E}=-e E_{z} / m c$, which changes along the beam as the longitudinal electric field $E_{z}=E_{z}(\xi)$ changes with comoving position.

The betatron frequency of an electron may be expanded for small relative energy deviations with respect to the mean slice energy $|\delta \gamma / \bar{\gamma}| \ll 1$ (overlined quantities refer to slice-averaged quantities), such that $\omega_{\beta} \simeq \bar{\omega}_{\beta}(1-\delta \gamma / 2 \bar{\gamma})$, where $\bar{\omega}_{\beta}$ is the mean slice betatron frequency. Hence, expressing the phase advance of an individual electron in terms of the mean slice phase advance,

$$
\varphi(t)=\bar{\varphi}(t)\left[1-\frac{\delta \gamma}{2 \bar{\gamma}_{0}} \frac{\overline{\omega_{\beta}}(t)}{\overline{\omega_{\beta, 0}}}\right]
$$

where $\bar{\varphi}=2\left(\overline{\omega_{\beta, 0}} / \overline{\omega_{\beta}}-1\right) / \epsilon$ is the mean slice phase advance, $\overline{\omega_{\beta, 0}}=\omega_{p} / \sqrt{2 \bar{\gamma}_{0}}$ the initial betatron frequency and $\overline{\omega_{\beta}}=\overline{\omega_{\beta, 0}} / \sqrt{1+\epsilon \overline{\omega_{\beta, 0}} t}$ the mean slice betatron frequency. It should be noted that the mean slice betatron frequency is time-dependent owing to a finite relative energy change per betatron cycle $\epsilon=\mathcal{E}\left(\bar{\gamma}_{0} \overline{\omega_{\beta, 0}}\right)^{-1}=$ $-\sqrt{2 / \bar{\gamma}_{0}} E_{z} / E_{0}$, with $E_{0}=\omega_{p} m c / e$. Equation (5) implies that electrons with differing energy within a slice acquire a differing phase advance, which leads to the phase-mixing of the electron betatron oscillations. This is illustrated in Fig. 1, in which the mean slice phase advance $\bar{\varphi}$ is depicted in gray. The deviated phase advance $\varphi_{ \pm}$according to Eq. (5) for electrons with an energy deviation of $\pm \delta \gamma$ is shown in orange. For this illustration, the parameters are chosen as $\epsilon=0.3$ and $\delta \gamma=0.3 \bar{\gamma}_{0}$. Here, the nonlinearity of the lines for $\bar{\varphi}$ and $\varphi_{ \pm}$is owing to a finite energy change $\epsilon \neq 0$ and the difference between $\bar{\varphi}$ and $\varphi_{ \pm}$is resulting from a finite energy deviation $\delta \gamma \neq 0$. The difference between the phase advance of electrons with differing energy grows as the mean slice phase advance $\bar{\varphi}$ evolves, and as the ratio $\overline{\omega_{\beta}}(t) / \overline{\omega_{\beta, 0}}$ increases [compare Eq. (5)]. If the phaseadvance difference is comparable to $\pi$, the betatron oscillations of the electrons are at opposite phase. For a large set of electrons with a finite energy distribution, the betatron phases become completely mixed. As shown within this work, this phase mixing damps the hose instability.

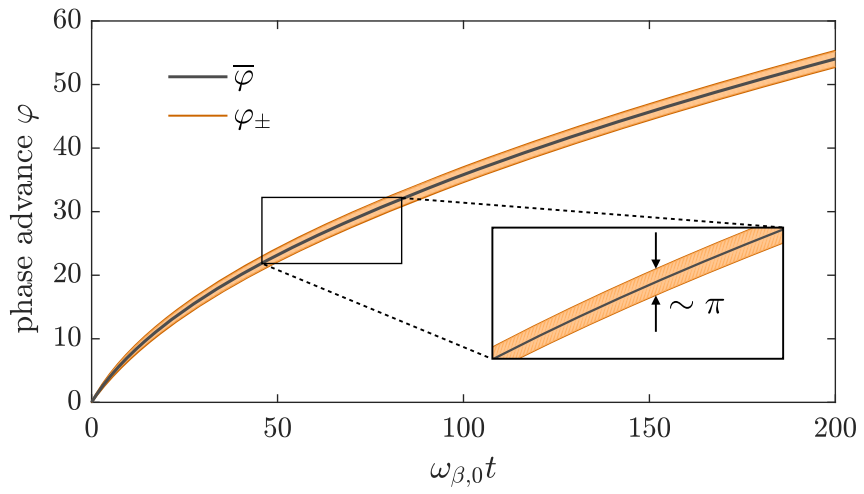

FIG. 1. Illustration of the phase advance of electrons within a beam slice. The gray curve depicts the mean slice phase advance $\bar{\varphi}$ and the orange curves illustrate the phase advance $\varphi_{ \pm}$ according to Eq. (5) for electrons with an energy deviation of $\pm \delta \gamma$ (for this illustration, $\epsilon=0.3$ and $\delta \gamma=0.3 \bar{\gamma}_{0}$ ). 


\section{B. Beam-centroid equation}

The beam centroid $X_{b}$ at a given comoving position $\xi$ is computed from Eq. (4). This is done by averaging the transverse position of electrons within each beam slice with respect to an initial phase-space distribution $f_{0}\left(x_{0}, p_{x, 0}, \gamma_{0}\right)$, that is $X_{b}(\xi, t)=\int x f_{0} \mathrm{~d} x_{0} \mathrm{~d} p_{x, 0} \mathrm{~d} \gamma_{0}$, where the distribution function is normalized, $\int f_{0} \mathrm{~d} x_{0} \mathrm{~d} p_{x, 0} \mathrm{~d} \gamma_{0}=1$. The initial transverse offset and momentum in a slice are hereby assumed to not to be correlated with energy, such that $f_{0}$ is separable $f_{0}=f_{\perp}\left(x_{0}, p_{x, 0}\right) f_{\gamma}\left(\gamma_{0}\right)$. The distribution $f_{\perp}\left(x_{0}, p_{x, 0}\right)$ is arbitrary, with the assumption that $f_{\perp}=0$ outside the channel, and the mean values are given by $\overline{x_{0}}=X_{b, 0}$ and $\overline{p_{x, 0}}=m c U_{b, 0}$ (i.e., $U_{b, 0}=\overline{p_{x, 0}} / m c$ ). We consider a Gaussian distributed energy, i.e., $f_{\gamma}=$ $\left(\sqrt{2 \pi} \sigma_{\gamma}\right)^{-1} \exp \left(-\delta \gamma^{2} / 2 \sigma_{\gamma}^{2}\right)$. The average with respect to the initial transverse phase space distribution and the Gaussian energy distribution, neglecting the variation of $A$ owing to $\delta \gamma$, yields

$$
\begin{aligned}
X_{b}(\xi, t) \simeq & \left\{X_{b, 0}(\xi) \cos [\bar{\varphi}(\xi, t)]+\frac{c U_{b, 0}(\xi)}{\bar{\gamma}_{0}(\xi) \overline{\omega_{\beta, 0}}(\xi)} \sin [\bar{\varphi}(\xi, t)]\right\} \bar{A}(\xi, t) \exp \left(-\frac{\Delta \gamma^{2} \bar{\alpha}(\xi, t)^{2}}{2}\right) \\
& +\overline{\omega_{\beta, 0}}(\xi) \int_{0}^{t} \bar{A}(\xi, t) \bar{A}\left(\xi, t^{\prime}\right) \exp \left(-\frac{\Delta \gamma^{2}\left[\bar{\alpha}(\xi, t)^{2}-\bar{\alpha}\left(\xi, t^{\prime}\right)^{2}\right]}{2}\right) \sin \left[\bar{\varphi}(\xi, t)-\bar{\varphi}\left(\xi, t^{\prime}\right)\right] X_{p}\left(\xi, t^{\prime}\right) \mathrm{d} t^{\prime}
\end{aligned}
$$

where $\Delta \gamma=\sigma_{\gamma} / \bar{\gamma}_{0}$ is the initial relative energy spread, $\bar{A}(\xi, t)=\left[\bar{\gamma}_{0}(\xi) / \bar{\gamma}(\xi, t)\right]^{1 / 4}$ the betatron amplitude and where $\bar{\alpha}(\xi, t)=\bar{\varphi}(\xi, t) \overline{\omega_{\beta}}(\xi, t) / 2 \overline{\omega_{\beta, 0}}(\xi)$. A term resulting from the $\delta \gamma$-dependence of the amplitude of the second term in equation (4) scales as $\bar{\alpha} \Delta \gamma^{2} \exp \left(-\bar{\alpha}^{2} \Delta \gamma^{2} / 2\right)$, and was neglected since $\Delta \gamma \ll 1$.

Equation (6) describes the centroid of a beam which is subject to hosing. The homogeneous part corresponds to a harmonic beam centroid oscillation with an amplitude which is modulated by the beam energy change and by an exponential damping owing to a finite uncorrelated energy spread. In the inhomogeneous part, the beam centroid is driven by the channel centroid $X_{p}$, which, through Eq. (1), couples the oscillation of individual beam slices with one another and thereby leads to hosing. The $\xi$-dependence of the Green's function in Eq. (6) implies that the coupling between individual slices is disrupted if their phase advance differs from the resonant coupling mediated via the plasma channel. For early times, Eqs. (1) and (6) recover known results for hosing in the blowout regime [14]. However, for greater times, Eq. (6) shows that the energy evolution, the energy chirp and the uncorrelated energy spread can have a substantial impact onto the hose instability, suggesting that hosing can be mitigated.

The differential form of Eq. (6) is given by

$$
\frac{\partial^{2} X_{b}}{\partial t^{2}}+\Lambda(\xi, t) \frac{\partial X_{b}}{\partial t}+\Omega(\xi, t)^{2}\left(X_{b}-X_{p}\right)=0,
$$

where

$$
\begin{gathered}
\Lambda(\xi, t)=\frac{\overline{\omega_{\beta}}(\xi, t)^{2}}{\overline{\omega_{\beta, 0}}(\xi)}\left(\epsilon(\xi)+\kappa_{1}(\xi, t) \Delta \gamma^{2}\right), \\
\Omega(\xi, t)^{2}=\overline{\omega_{\beta}}(\xi, t)^{2}\left(1+\kappa_{2}(\xi, t) \Delta \gamma^{2}\right),
\end{gathered}
$$

and where $\kappa_{1}=\left(\overline{\omega_{\beta}} / \overline{\omega_{\beta, 0}}-\left(\overline{\omega_{\beta}} / \overline{\omega_{\beta, 0}}\right)^{2}\right) / \epsilon$, and $\kappa_{2}=$ $\left(\overline{\omega_{\beta}} / \overline{\omega_{\beta, 0}}\right)^{4} / 2-\left(\overline{\omega_{\beta}} / \overline{\omega_{\beta, 0}}\right)^{3} / 4$. Terms scaling as $\mathcal{O}\left(\Delta \gamma^{4}\right)$ and $\mathcal{O}\left(\epsilon^{2}\right)$ were hereby neglected. Equations (6) and (7) hold for any relativistic beam within a blowout with small $|\epsilon|=\sqrt{2 / \bar{\gamma}_{0}}\left|E_{z} / E_{0}\right| \ll 1$ and $\Delta \gamma \ll 1$.

\section{Qualitative analysis of the beam-centroid equation}

The coefficients $\Lambda$ and $\Omega$ can have multiple effects. A finite friction term $\Lambda$ results in a damping of the beam centroid oscillation if $\Lambda>0$ or an amplification if $\Lambda<0$. If $\Omega$ is time-independent but $\xi$-dependent, the betatron oscillations of various slices are developing a decoherence with respect to each other. Equivalently, for an $\Omega$ which is initially not $\xi$-dependent but features a varying timedependence along $\xi$, a detuning of the oscillation phases of various slices develops over time.

A linear expansion of (8) and (9) yields $\Lambda \simeq \overline{\omega_{\beta, 0}}(\epsilon+$ $\left.\Delta \gamma^{2} \overline{\omega_{\beta, 0}} t / 2\right)$ and $\Omega^{2} \simeq{\overline{\omega_{\beta, 0}}}^{2}\left(1+\Delta \gamma^{2} / 4-\epsilon \overline{\omega_{\beta, 0}} t\right)$, allowing for a qualitative assessment of the implications of Eq. (7) onto the hose instability. For no energy change and no uncorrelated and correlated energy spread, i.e., for $\epsilon=0, \Delta \gamma=0$ and $\overline{\omega_{\beta, 0}}(\xi)=\omega_{\beta}$, respectively, Eq. (7) recovers the original monoenergetic beam-centroid equation (2) used in previous studies [10-14]. For a finite energy gain/loss $(\epsilon \neq 0)$, a finite uncorrelated energy spread $(\Delta \gamma \neq 0)$ and/or an initial chirp $\left(\partial_{\xi} \overline{\omega_{\beta, 0}} \neq 0\right)$, the following conclusions can be drawn.

The centroid amplitude of a beam with a finite uncorrelated energy spread $(\Delta \gamma \neq 0)$, is damped over time, since $\Lambda \sim \Delta \gamma^{2} \overline{\omega_{\beta, 0}} t>0$. For slices within the beam with $\epsilon>0$, i.e., in regions in which electrons are being accelerated, the term $\Lambda$ implies an increased damping of the beam centroid for all times. In slices for which $\epsilon<0$, the centroid is initially slightly amplified before it is progressively damped. The initial amplification of the oscillation 
amplitude is a result of the relativistic mass loss of electrons which are being decelerated [compare with Eq. (3)]. At greater times, the damping from the finite energy spread dominates. Additionally, it can be seen that for an initial chirp $\left(\partial_{\xi} \overline{\omega_{\beta, 0}} \neq 0\right)$, and/or for $\xi$-dependence of the energy change $\left(\partial_{\xi} \epsilon \neq 0\right)$, the centroid oscillation frequency $\Omega$ initially features, or develops a $\xi$-dependence, respectively. As shown in the following more quantitatively, this $\xi$ dependence leads to a decoherence of the individual sliceoscillations which disrupts the resonant coupling via the plasma and thereby results in a saturation of hosing.

In the following, we use a two-particle model to allow for a more quantitative interpretation of the new beam centroid equation in terms of the mitigation times from the above described effects.

\section{INTERPRETATION USING A TWO-PARTICLE BEAM}

We use a two-particle (i.e., a two-slice) model to analytically investigate the physical predictions of Eqs. (1) and (6) in terms of the timescales for the hosing mitigation. In the two particle model, the beam consists of two slices with centroids $X_{b, 1}(t)$ at $\xi_{1}$ and $X_{b, 2}(t)$ at $\xi_{2}$. The two slices are initially at the same oscillation phase $\bar{\varphi}\left(\xi_{1}, t=0\right)=\bar{\varphi}\left(\xi_{2}, t=0\right)=0$.

The first slice is unaffected by the hose instability, i.e., $X_{p}\left(\xi \leq \xi_{1}\right)=0$, and the general equation for the beam centroid evolution, Eq. (6), therefore yields for the centroid of the first slice

$X_{b, 1}(t)=\hat{X}_{b, 1} \bar{A}\left(\xi_{1}, t\right) \exp \left(-\frac{\Delta \gamma^{2} \bar{\alpha}\left(\xi_{1}, t\right)^{2}}{2}\right) \cos \left[\bar{\varphi}\left(\xi_{1}, t\right)\right]$

where $\hat{X}_{b, 1}$ is the initial oscillation amplitude. The centroid of the slice at $\xi_{1}$ therefore oscillates with an amplitude which is time-dependent according to $\bar{A}\left(\xi_{1}, t\right)$ and according to the exponential damping from the finite energy spread. The oscillation frequency is time-dependent as a result of the nonlinear time dependence of $\bar{\varphi}$.

As implied by Eq. (1), the oscillation of the first slice induces a deviation of the blowout channel centroid. At the comoving position of the trailing slice the channel centroid deviation is

$$
X_{p}\left(\xi_{2}, t\right)=I_{\xi_{1}}^{\xi_{2}} X_{b, 1}(t)
$$

where $I_{\xi_{1}}^{\xi_{2}}$ is a blowout-geometry-dependent constant. The channel centroid deviation along $\xi$ feeds back into the timeevolution of the centroid oscillation of the second slice at $\xi_{2}$ according to Eq. (6), such that

$$
\begin{aligned}
X_{b, 2}(t)= & \bar{A}\left(\xi_{2}, t\right) \exp \left[-\frac{\Delta \gamma^{2} \bar{\alpha}\left(\xi_{2}, t\right)^{2}}{2}\right]\left\{\hat{X}_{b, 2} \cos \left[\bar{\varphi}\left(\xi_{2}, t\right)\right]\right. \\
& \left.+\overline{\omega_{\beta, 0}}\left(\xi_{2}\right) \int_{0}^{t} \bar{A}\left(\xi_{2}, t^{\prime}\right) \exp \left[\frac{\Delta \gamma^{2} \bar{\alpha}\left(\xi_{2}, t^{\prime}\right)^{2}}{2}\right] \sin \left[\bar{\varphi}\left(\xi_{2}, t\right)-\bar{\varphi}\left(\xi_{2}, t^{\prime}\right)\right] X_{p}\left(\xi_{2}, t^{\prime}\right) \mathrm{d} t^{\prime}\right\}
\end{aligned}
$$

The oscillation of the trailing slice is driven by the oscillation of first slice through the coupling by the plasma channel centroid. Equations (10), (11), and (12) in combination serve as a basis for the derivations of the decoherence times from a differential energy change, from an initial chirp and from an uncorrelated energy spread, detailed below.

\section{A. Decoherence time from a differential energy change}

We start by determining the timescale for the hosing saturation by virtue of a finite differential energy change along the beam $\left(\partial_{\xi} \epsilon \neq 0\right)$. To isolate this effect, we assume no initial chirp, no uncorrelated energy spread $(\Delta \gamma=0)$, and that the particles have an energy on the order of the initial energy, i.e., $\gamma \sim \gamma_{0}$, such that $\bar{A}(\xi, t) \approx 1$. These assumptions, together with Eqs. (10), (11), and (12), yield for the centroid of the second slice

$$
X_{b, 2}(t) \simeq \hat{X}_{b, 2} \cos \left[\bar{\varphi}\left(\xi_{2}, t\right)\right]+\hat{X}_{b, 1} I_{\xi_{1}}^{\xi_{2}} I_{\epsilon}(t),
$$

with the time-dependent amplitude integral

$I_{\epsilon}(t)=\overline{\omega_{\beta, 0}} \int_{0}^{t} \sin \left[\bar{\varphi}\left(\xi_{2}, t\right)-\bar{\varphi}\left(\xi_{2}, t^{\prime}\right)\right] \cos \left[\bar{\varphi}\left(\xi_{1}, t^{\prime}\right)\right] \mathrm{d} t^{\prime}$.

It should be noted that $I_{\epsilon}(t)$ is the only term in Eq. (13) with time-dependent amplitude. We use $|\epsilon|=\left|\sqrt{2 / \bar{\gamma}_{0}} E_{z} / E_{0}\right| \ll 1$ to expand the phase advance

$$
\begin{aligned}
\bar{\varphi}(\xi, t) & =\frac{2\left[\sqrt{1+\epsilon(\xi) \overline{\omega_{\beta, 0}}} t-1\right]}{\epsilon(\xi)} \\
& \simeq \overline{\omega_{\beta, 0}} t-\frac{{\overline{\omega_{\beta, 0}}}^{2} t^{2} \epsilon(\xi)}{4} .
\end{aligned}
$$


By use of this expansion, the integral (14) yields

$$
\begin{aligned}
I_{\epsilon}(t) \simeq & \sqrt{\frac{\pi}{2 \Delta \epsilon}}\left[\sin \left[\bar{\varphi}\left(\xi_{2}, t\right)\right] F_{c}\left(\sqrt{\frac{\Delta \epsilon}{2 \pi}} \overline{\omega_{\beta, 0}} t\right)\right. \\
& \left.-\cos \left[\bar{\varphi}\left(\xi_{2}, t\right)\right] F_{s}\left(\sqrt{\frac{\Delta \epsilon}{2 \pi}} \overline{\omega_{\beta, 0}} t\right)\right],
\end{aligned}
$$

where $|\epsilon| \ll 1$ was exploited in order to simplify the expression. Here $F_{s}$ and $F_{c}$ are the Fresnel integrals and $\Delta \epsilon=\left|\epsilon\left(\xi_{1}\right)-\epsilon\left(\xi_{2}\right)\right|$ is the difference of the normalized rates of energy change between the slices. The oscillations of the sine and cosine functions occur on timescales much shorter than the variations of the Fresnel integrals for $\Delta \epsilon \ll 1$. Averaging $I_{\epsilon}{ }^{2}$ over one period in a slowly varying amplitude approximation yields the time-dependence

$$
\left\langle I_{\epsilon}(t)^{2}\right\rangle \sim F_{c}^{2}\left(\sqrt{\frac{\Delta \epsilon}{2 \pi}} \overline{\omega_{\beta, 0}} t\right)+F_{s}^{2}\left(\sqrt{\frac{\Delta \epsilon}{2 \pi}} \overline{\omega_{\beta, 0}} t\right) .
$$

In Fig. 2, the temporal evolution of $\sqrt{\left\langle I_{\epsilon}(t)^{2}\right\rangle}$ is plotted in arbitrary units so as to visualize that the centroid oscillation amplitude of the second slice initially increases due to a resonant coupling of the two slices. However, when the phase difference between the two slices becomes significant, $X_{b}\left(\xi_{2}\right)$ reaches a global maximum at

$$
\overline{\omega_{\beta, 0}} t_{\mathrm{d}, \epsilon} \simeq \sqrt{\frac{3 \pi}{\Delta \epsilon}},
$$

and subsequently saturates at a smaller amplitude. Equation (17) therefore specifies a measure for the hosing-mitigation time from a differential energy change along the beam. This result is valid for arbitrary coefficients $\mathcal{C}_{p}(\xi), \mathcal{C}_{d}(\xi)$, and $\mathcal{C}_{b}(\xi)$ in Eq. (1) and is in fundamental contrast with previous models for the hose instability, which predict steadily growing amplitudes until beam breakup.

We compare the hosing mitigation time from differential energy change $t_{\mathrm{d}, \epsilon}$ with the drive beam energy depletion time. The energy of the drive beam is depleted at $t_{\mathrm{dp}}=$ $1 /\left(\overline{\omega_{\beta, 0}} \hat{\epsilon}\right)$, where $\hat{\epsilon}=-\sqrt{2 / \bar{\gamma}_{0}} \hat{E}_{d} / E_{0}$ and $\hat{E}_{d}$ denotes the

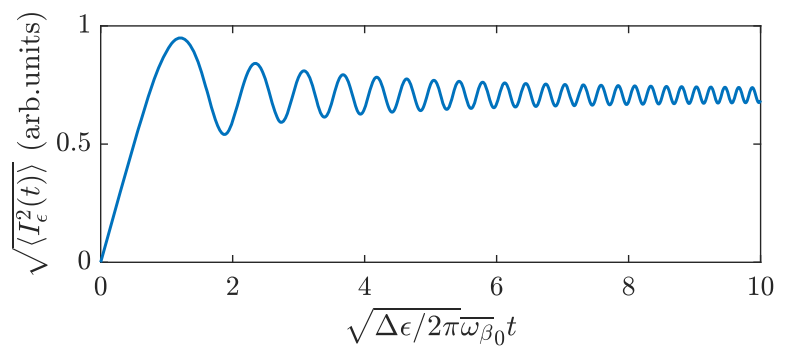

FIG. 2. Depiction of time-dependent amplitude of the second slice according to Eq. (17). maximum longitudinal field in the drive beam region. Hence, decoupling between the two slices occurs before pump depletion if $\Delta \epsilon / \hat{\epsilon}>3 \pi \hat{\epsilon}$. Because $|\hat{\epsilon}| \ll 1$, and since $\Delta \epsilon / \hat{\epsilon}$ ranges from zero to unity along any drive beam, the two particle model suggests that slices within the beam in PWFAs are decoupled significantly before depletion.

The normalized relative acceleration rate $\epsilon$ is related to key experimental PWFA parameters, such as the beam peak current and the beam energy as follows. The longitudinal field within the beam region can be approximated by $E_{z}(\xi) / E_{0} \simeq \sqrt{I_{b}(\xi) / I_{A}}$, where $I_{b}(\xi)$ is the beam current and $I_{A} \simeq 17 \mathrm{kA}$ is the Alfvén current [26,27]. Hence, $\epsilon(\xi) \simeq-\sqrt{2 I_{b}(\xi) /\left(I_{A} \bar{\gamma}_{0}\right)}$, and for experimental parameters which resemble those in the Facility for Advanced Accelerator Experimental Tests (FACET) [28,29], for instance, yield $\hat{\epsilon} \approx 0.007$. This, together with the argumentation above indicates that the growth of the hose instability stops well before energy depletion in typical PWFA scenarios and possibly justifies why hosing was not detected in previous experiments [3,5]. For completeness, it should be noted that the drive beams in FACET had a transverse extent comparable to the blowout radius and therefore, in addition to the above elaborated decoherence effect, were undergoing a head-to-tail decoherence owing to a variation of the focusing fields in the partial blowout along the beam [19].

\section{B. Decoherence time from an initial chirp}

We investigate the decoherence time owing to an initial linear energy chirp of a beam,

$$
\chi=\frac{1}{\gamma_{b} k_{p}} \frac{\partial \bar{\gamma}}{\partial \xi}
$$

where $\gamma_{b}$ is the beam-averaged energy. To do so, we isolate this effect by regarding particles with an energy comparable to the initial energy i.e., $\gamma \sim \gamma_{0}$, such that $\bar{A}(\xi, t) \approx 1$, and by assuming no uncorrelated energy spread $(\Delta \gamma=0)$ and no energy change $(\epsilon=0)$. Using these assumptions, Eqs. (10), (11), and (12) yield

$$
X_{b, 2}(t) \simeq \hat{X}_{b, 2} \cos \left[\overline{\omega_{\beta, 0}}\left(\xi_{2}\right) t\right]+\hat{X}_{b, 1} I_{\xi_{1}}^{\xi_{2}} I_{\chi}(t),
$$

with the time-dependent amplitude term

$I_{\chi}(t)=\overline{\omega_{\beta, 0}}\left(\xi_{2}\right) \int_{0}^{t} \sin \left[\overline{\omega_{\beta, 0}}\left(\xi_{2}\right)\left(t-t^{\prime}\right)\right] \cos \left[\overline{\omega_{\beta, 0}}\left(\xi_{1}\right) t^{\prime}\right] \mathrm{d} t^{\prime}$.

Evaluation of this integral yields

$I_{\chi}(t)=\frac{\overline{\omega_{\beta, 0}}\left(\xi_{2}\right)^{2}\left\{\cos \left[\overline{\omega_{\beta, 0}}\left(\xi_{2}\right) t\right]-\cos \left[\overline{\omega_{\beta, 0}}\left(\xi_{1}\right) t\right]\right\}}{\left[\overline{\omega_{\beta, 0}}\left(\xi_{1}\right)-\overline{\omega_{\beta, 0}}\left(\xi_{2}\right)\right]\left[\overline{\omega_{\beta, 0}}\left(\xi_{1}\right)+\overline{\omega_{\beta, 0}}\left(\xi_{2}\right)\right]}$.

Hence, the second slice is driven antiresonantly at 


$$
t_{\mathrm{d}, \chi}=\frac{\pi}{\left|\Delta \overline{\omega_{\beta, 0}}\right|},
$$

where $\Delta \overline{\omega_{\beta, 0}}=\overline{\omega_{\beta, 0}}\left(\xi_{1}\right)-\overline{\omega_{\beta, 0}}\left(\xi_{2}\right)$. The integral $I_{\chi}(t)$ shows a beating since, after time $3 / 2 \times t_{\mathrm{d}, \chi}$, the second slice is driven resonantly again, and so forth. This is in contrast to a continuous beam with an infinite number of slices, for which the amplitude of $I_{\chi}(t)$ decays. For the situation with no chirp, $\Delta \overline{\omega_{\beta, 0}} \rightarrow 0$, implies $t_{\mathrm{d}, \chi} \rightarrow \infty$ for the decoherence time and implies that $I_{\chi}(t)$ is growing linearly.

The following considerations connect the decoherence time of the two-particle model with the decoherence time of a continuous beam with a linear energy chirp. Expanding the frequency difference of the slice betatron oscillations at the mean beam energy $\gamma_{b}$ for small $\Delta \bar{\gamma}$ with $\overline{\gamma_{0}}\left(\xi_{1}\right)=$ $\gamma_{b}+\Delta \bar{\gamma} / 2$ and $\overline{\gamma_{0}}\left(\xi_{2}\right)=\gamma_{b}-\Delta \bar{\gamma} / 2$ yields

$$
\Delta \overline{\omega_{\beta, 0}} \simeq-\frac{\omega_{\beta, b}}{2 \gamma_{b}} \Delta \bar{\gamma},
$$

where $\omega_{\beta, b}=\omega_{p} / \sqrt{2 \gamma_{b}}$ is the mean beam betatron frequency.

Combining Eqs. (18), (21), and (22) then yields

$$
\omega_{\beta, b} t_{\mathrm{d}, \chi} \simeq \frac{2 \pi}{|\chi| k_{p} \Delta \xi}
$$

where $\Delta \xi$ is the spacing between the two slices. Equation (23) provides an estimate of the timescale for the mitigation of the hose instability owing to an initial linear energy chirp $\chi$. However, it should be noted that the mitigation time owing to an energy chirp of a continuous beam generally cannot be accurately described by a twoparticle model [30] and rigorously also depends on the wake response, here, on the blowout geometry. The mitigation of hosing owing to energy chirps is analogous to Balakin-Novokhatsky-Smirnov damping [16] in conventional accelerators.

\section{Decoherence time from an uncorrelated energy spread}

Additionally, Eq. (6) implies that the amplitude of the $X_{b}$ oscillations are damped exponentially for a finite uncorrelated energy spread $\Delta \gamma$. Assuming no initial correlated energy spread $(\chi=0)$ and that slices are not close to depletion $[\bar{A}(\xi, t) \approx 1]$, the centroid amplitude of individual slices, according to Eq. (6), is reduced by half an e-folding if $\Delta \gamma^{2} \bar{\alpha}(\xi, t)^{2}=1$. Since $\bar{\alpha}=\bar{\varphi} \bar{\omega}_{\beta} / 2 \overline{\omega_{\beta, 0}}=$ $\left[1-\left(1+\epsilon \overline{\omega_{\beta, 0}} t\right)^{-1 / 2}\right) / \epsilon$, this is the case at the decoherence time

$$
\overline{\omega_{\beta, 0}} t_{\mathrm{d}, \Delta \gamma}=\frac{2 \Delta \gamma-\epsilon}{(\Delta \gamma-\epsilon)^{2}} .
$$

The damping of the hose instability is significant, if the decoherence time is on the order of (or smaller than) the depletion time $t_{\mathrm{d}, \Delta \gamma} \lesssim t_{\mathrm{dp}}=1 / \hat{\epsilon} \overline{\omega_{\beta, 0}}$. This is generally true near to the peak electric field, where $|\epsilon| \approx \hat{\epsilon}$.

In the conservative limit of no change of the mean slice energy, $\epsilon \rightarrow 0$, the decoherence time simplifies to

$$
\overline{\omega_{\beta, 0}} t_{\mathrm{d}, \Delta \gamma} \simeq \frac{2}{\Delta \gamma} .
$$

Equation (25) specifies a timescale for the damping of the amplitude of the centroid oscillations owing to a finite energy spread in the conservative scenario of no energy change. Therefore, $t_{\mathrm{d}, \Delta \gamma} \lesssim t_{\mathrm{dp}}$ if $\Delta \gamma \gtrsim 2 \hat{\epsilon}$.

For the typical parameters of FACET, where $\hat{\epsilon} \approx 0.007$, a subpercent-level energy spread already significantly contributes to the mitigation of hosing. Moreover, it should be noted that if $t_{\mathrm{d}, \epsilon} \lesssim t_{\mathrm{d}, \Delta \gamma}$, the exponential damping of $X_{b}$ due to the uncorrelated energy spread becomes substantial since $X_{b}$ stops growing owing to finite $\partial_{\xi} \epsilon$.

\section{COMPARISON TO PARTICLE-IN-CELL SIMULATIONS}

We present simulation results obtained with the threedimensional (3D) quasistatic PIC code HIPACE [20] which demonstrate the effectiveness of the mitigation mechanisms for beam parameters close to those used in current PWFA experiments and to those foreseen in future PWFA experiments at major facilities.

In the first set of simulations we investigate hosing for Gaussian beams with varying beam energy and peak current. The second simulation study investigates the effect of a varying uncorrelated energy spread of a beam with a triangular current profile. These setups feature greater beam energies than in the example presented in Ref. [15] and differing beam peak currents, current profiles, hosing seeds, and initial energy spreads.

\section{A. Hosing mitigation for varying beam energies}

We consider tilted Gaussian electron beams with a varying initial beam energy and differing beam peak currents. The beam in case $\mathrm{C}_{1 \mathrm{a}}$ has an energy of $5 \mathrm{GeV}$ and $5 \mathrm{kA}$ peak current, beam $\mathrm{C}_{1 \mathrm{~b}}$ has an energy of $10 \mathrm{GeV}$ and $10 \mathrm{kA}$ peak current (corresponding to FACET II parameters [31]) and case $\mathrm{C}_{1 \mathrm{c}}$ features $25 \mathrm{GeV}$ and $23 \mathrm{kA}$ (corresponding to FACET parameters [28]), respectively (see Table I). The dimensions of the Gaussian beams are identical to the ones used for the simulations in [15] with a transverse size of $k_{p} \sigma_{x}=k_{p} \sigma_{y}=0.1$, and length of $k_{p} \sigma_{z}=1.0$. The beams with the peak current located at $k_{p} \xi=0.0$ drive a plasma wave in the blowout regime in a homogeneous plasma target with density $n_{0}$. The initial centroid along the beam is given by $k_{p} X_{b, 0}(\xi)=0.001 \times$ $\left[k_{p}\left(\xi-\xi_{0}\right)\right] \Theta\left[k_{p}\left(\xi-\xi_{0}\right)\right]$, where $\Theta(x)$ is the Heavisidestep function. Hence, the beam tilt is introduced from $k_{p} \xi_{0}=-1.0$, close to the beam heads. We investigate the 
TABLE I. Beam parameters for the considered cases.

\begin{tabular}{lccc}
\hline \hline & Case $\mathrm{C}_{1 \mathrm{a}}$ & Case $\mathrm{C}_{1 \mathrm{~b}}$ & Case $\mathrm{C}_{1 \mathrm{c}}$ \\
\hline Beam energy $\bar{\gamma}_{0}$ & 9785 & 19569 & 48923 \\
Beam peak current $\hat{I}_{b} / I_{A}$ & 0.29 & 0.58 & 1.35 \\
\hline \hline
\end{tabular}

effect from the intrinsic beam energy change and isolate this effect by using beams with no initial correlated $(\chi=0)$ or uncorrelated energy spread $(\Delta \gamma=0)$. The parameters used here are close to the experimental parameters of current facilities [28,31] or of concepts for future facilities [29].

The simulations are performed with the 3D quasistatic PIC code HIPACE [20] using an updated field solver which computes all electromagnetic field components. The dimensions of the simulation boxes for the different cases vary due to a different blowout radius and were chosen as $9 \times 10 \times 10 k_{p}^{-3}$ for cases $\mathrm{C}_{1 \mathrm{a}}$ and $\mathrm{C}_{1 \mathrm{~b}}$, and $9 \times 12 \times 12 k_{p}^{-3}$ for case $\mathrm{C}_{1 \mathrm{c}}$. The number of cells is $512 \times 384 \times 384$ for cases $\mathrm{C}_{1 \mathrm{a}}$ and $\mathrm{C}_{1 \mathrm{~b}}$ and $512 \times 448 \times 448$ for case $\mathrm{C}_{1 \mathrm{c}}$. The time step is adjusted dynamically during the simulation and spans from $\omega_{p} \Delta t_{\text {init }}=9.0$ at the beginning of the simulation to $\omega_{p} \Delta t_{\text {fin }}=3.6$ at the end of the simulation $\left(\omega_{p} t_{\max }=2.3 \times 10^{4}\right)$ for case $\mathrm{C}_{1 \mathrm{a}}$. For case $\mathrm{C}_{1 \mathrm{~b}}$, the time step ranged from $\omega_{p} \Delta t_{\text {init }}=13.0, \omega_{p} \Delta t_{\text {fin }}=6.3$, with $\omega_{p} t_{\max }=2.7 \times 10^{4}$. For case $\mathrm{C}_{1 \mathrm{c}}$, the time step ranged from $\omega_{p} \Delta t_{\text {init }}=20.0$ to $\omega_{p} \Delta t_{\text {fin }}=7.3$ with $\omega_{p} t_{\text {max }}=$ $4.5 \times 10^{4}$. In all the simulations, the plasma is modelled with 4 particles per cell and the beams with 18 particles per cell using a quadratic charge interpolation.

The results for the beam centroid evolution at the tail of the beams at $k_{p} \xi=4.0$ are depicted in Fig. 3(a) for the three cases. It can be seen that the beam centroid oscillation amplitude for all three cases initially increases exponentially as predicted by previous models [14]. However, the growth of the hose instability stops after some oscillation periods and the beam centroid amplitudes subsequently reach a maximum. This is owing to the evolving decoherence of the oscillations of individual beam slices, which lead to a saturation of the hose instability.

The longitudinal field can be approximated by $E_{z} / E_{0} \simeq$ $\sqrt{I_{b} / I_{A}}$ within the beam region [26,27], such that the normalized rate of energy change per betatron cycle, $\epsilon=$ $-\sqrt{2 / \bar{\gamma}_{0}} E_{z} / E_{0}$, can be estimated by $\epsilon \simeq-\sqrt{2 I_{b} /\left(I_{A} \bar{\gamma}_{0}\right)}$. This implies that $\epsilon(\xi)$ is comparable for all beams. Hence, the mitigation of the hose instability is expected to occur on the same time scale (when normalized to the individual initial beam betatron frequencies) for all three cases. Assuming for simplicity that the difference of the acceleration rates $\Delta \epsilon$ for a slice at the tail and a driving slice closer to the beam head scales as $\Delta \epsilon \sim \hat{\epsilon}=\sqrt{2 \hat{I}_{b} /\left(I_{A} \bar{\gamma}_{0}\right)}$, where $\hat{I}_{b}$ is the beam peak current, we obtain $\Delta \epsilon \sim 0.007$

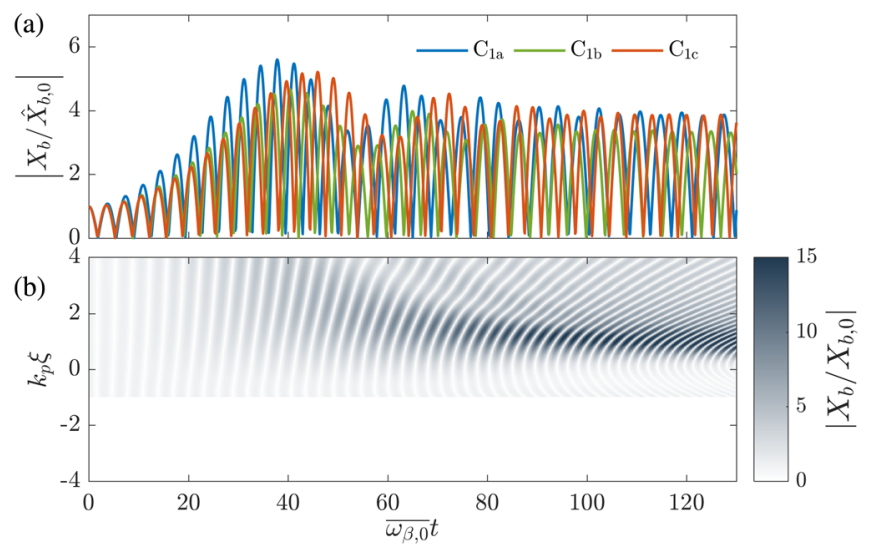

FIG. 3. (a) Evolution of beam centroid at the beam tails (at $k_{p} \xi=4.0$ ) for a beam with $5 \mathrm{GeV}$ and $5 \mathrm{kA}$, case $\mathrm{C}_{1 \mathrm{a}}$ (blue), for a beam with $10 \mathrm{GeV}$ and $10 \mathrm{kA}$, case $\mathrm{C}_{1 \mathrm{~b}}$ (green) and for a beam with $25 \mathrm{GeV}$ and $23 \mathrm{kA}$, case $\mathrm{C}_{1 \mathrm{c}}$ (red), obtained from PIC simulations. (b) Centroid evolution in $t$ and along $\xi$ for case $\mathrm{C}_{1 \mathrm{~b}}$ as retrieved from a PIC simulation.

for all three cases (compare Fig. 4). This yields $\overline{\omega_{\beta, 0}} t_{\mathrm{d}, \epsilon} \sim$ 37 for all cases [damping time given by Eq. (17)]. This simple estimate qualitatively agrees with the results shown in 3(a). The beam centroid oscillations stop growing on the same timescale $\left(\omega_{\beta, 0} t \sim 40\right)$ and saturate at comparable values for $t>t_{\mathrm{d}, \epsilon}$.

The subtle differences of the mitigation times for the different cases, shown in Fig. 3, come from the fact that $\epsilon(\xi)$ does not have precisely the same $\xi$-dependence along the beams for all the cases. The above used approximation $E_{z} / E_{0} \simeq \sqrt{I_{b} / I_{A}}$, which led to the assumption of an equal $\epsilon(\xi)$ for all cases, is strictly valid only for beams with a peak current exceeding $I_{A}[26,27]$. However, as shown in Fig. 4, the length of the blowout structure increases with increasing peak current and $\epsilon(\xi)$ features an enhanced slope towards the beam tail for lower beam currents when compared to cases with higher peak currents in the PIC simulations. Hence, the beam centroid amplitude the tails

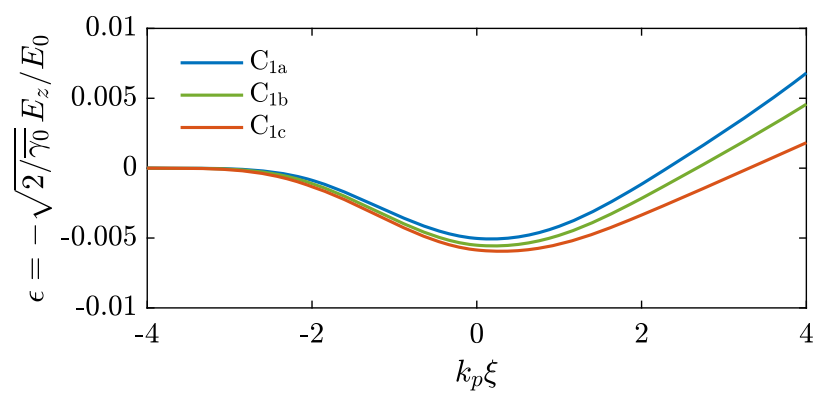

FIG. 4. The relative energy change per betatron cycle $\epsilon=$ $-\sqrt{2 / \bar{\gamma}_{0}} E_{z} / E_{0}$ as a function of the comoving coordinate $\xi$ for cases $\mathrm{C}_{1 \mathrm{a}}$ (blue), $\mathrm{C}_{1 \mathrm{~b}}$ (green), and $\mathrm{C}_{1 \mathrm{c}}$ (red) obtained from PIC simulations. 
reaches a maximum for $\mathrm{C}_{1 \mathrm{a}}$ earlier than for $\mathrm{C}_{1 \mathrm{~b}}$, and earlier for $\mathrm{C}_{1 \mathrm{~b}}$ than for $\mathrm{C}_{1 \mathrm{c}}$, respectively.

The evolution of $X_{b}$ for the complete beam is depicted in Fig. 3(b) for case $C_{1 b}$. The beam centroid initially grows in $t$ and along $\xi$. However, as soon as the oscillations of beam slices start to decohere with respect to each other, owing to a differing energy change $\left(\partial_{\xi} \epsilon \neq 0\right)$, the beam centroid stops growing along $\xi$. At the tail, $X_{b}$ reaches a maximum after about six oscillation periods [see Fig. 3(a), green curve] and saturates for greater $t$. This is in fundamental contrast to previous models which predict $X_{b}$ to grow steadily for increasing $\xi$. The decoherence time and connected hosing saturation time is different for different slices along the beam. This is owing to a variation of $\partial_{\xi} \epsilon(\xi), \mathcal{C}_{p}(\xi), \mathcal{C}_{b}(\xi)$, and $\mathcal{C}_{d}$ along the beam. Saturation of hosing takes place latest slightly behind the comoving position of maximum $E_{z}$ (e.g., at $k_{p} \xi=-0.20$ for $\mathrm{C}_{1 \mathrm{~b}}$ ), where $\partial_{\xi} \epsilon \approx 0$ (compare Fig. 4). This is due to the fact that slices behind the $E_{z}$ peak are driven coherently by slices ahead of the peak location for a greater amount of time than, e.g., slices at the tail. Yet, as the differential phase difference of centroid oscillations along the beam increases, Eq. (1) implies that the channel centroid is stopped being amplified and the beam centroid amplitude saturates also close to the $E_{z}$ peak. Hence, for times comparable to $t_{\mathrm{d}, \epsilon}$ or greater, the beam centroid oscillation amplitude does not grow steadily with increasing $\xi$, as predicted in current models. Instead, $X_{b}$ increases toward the region with minimum $\epsilon$, where decoherence takes place later and decreases for greater $\xi$.

In conclusion, the here presented simulations show that the intrinsic drive-beam energy evolution in PWFA leads to a mitigation of the hose instability independent of the initial beam energy and for other types of hosing seed than the ones regarded in Ref. [15]. Different to what is expected from previous models, we find that hosing does not grow steadily along $\xi$ but for $t \gtrsim t_{\mathrm{d}, \epsilon}$ increases towards the region with maximum $E_{z}$ and decreases for greater $\xi$. This observation is consistent with the predictions from the two particle model.

\section{B. Hosing mitigation for varying degrees of beam energy spread}

In this section we present results from 3D PIC simulations investigating the hosing of a drive beam with triangular current profile. This is in distinction to the previous examples of beams with Gaussian current profiles presented in [15] and above. We regard a beam with the parameters used in Ref. [14]. The beam has an energy of $\bar{\gamma}_{0}=55773$, and a transverse Gaussian density distribution with transverse dimensions $k_{p} \sigma_{x}=k_{p} \sigma_{y}=13.0 \times 10^{-3}$. The current profile is triangular with the current rising from zero at $k_{p} \xi=-0.8$ to the peak value of $\hat{I}_{b}=6.65 \mathrm{kA}$ at $k_{p} \xi=-0.53$. From this position, the current decreases linearly to zero at $k_{p} \xi=3.19$. The initial beam centroid is given by $X_{b, 0}(\xi)=4.17 \times 10^{-3} \times \xi \Theta\left(k_{p} \xi\right)$, hence, a tilt is introduced from position $k_{p} \xi=0$. The beam propagates through a flat-top plasma target with density $n_{0}$ and drives a plasma wave in the blowout regime. We investigate the hose instability for the case of no beam energy spread as in Ref. [14], and study how the degree of the initial relative uncorrelated energy spread $\Delta \gamma=\sigma_{\gamma} / \bar{\gamma}_{0}$ affects the hose instability. Case $\mathrm{C}_{2 \mathrm{a}}$ is without energy spread, $\Delta \gamma=0.0$, case $\mathrm{C}_{2 \mathrm{~b}}$ has a relative energy spread of $\Delta \gamma=0.005$, case $\mathrm{C}_{2 \mathrm{c}}$ has $\Delta \gamma=0.01$ and case $\mathrm{C}_{2 \mathrm{~d}}$ features $\Delta \gamma=0.02$.

The simulations are performed using the $3 \mathrm{D}$ quasistatic PIC code HIPACE [20]. The dimensions of the simulation box are $5 \times 9 \times 9 k_{p}^{-3}$ and the number of cells $512 \times 512 \times$ 512. The time step is adjusted dynamically during the simulation and spans from $\omega_{p} \Delta t_{\text {init }}=20.0$ at the beginning of the simulation to $\omega_{p} \Delta t_{\text {fin }}=6.0$ at the end of the simulation $\left(\omega_{p} t_{\max }=1.0 \times 10^{5}\right)$. Four particles per cell are used to model the plasma and 72 beam particles per cell are used for case $\mathrm{C}_{2 \mathrm{a}}$ and 16384 beam particles per cell for cases $\mathrm{C}_{2 \mathrm{~b}}, \mathrm{C}_{2 \mathrm{c}}$, and $\mathrm{C}_{2 \mathrm{~d}}$, using a quadratic charge interpolation scheme.

Figure 5(a) depicts the temporal evolution of the beam centroid at the tail of the beam $\left(k_{p} \xi=3.15\right)$ for all four cases. The beam centroid amplitude grows exponentially until $\overline{\omega_{\beta, 0}} t \sim 35$, in agreement with the result provided in Ref. [14]. However, we find that the growth rate for greater propagation distances is not exponential. Instead, the beam centroid reaches a maximum at $\overline{\omega_{\beta, 0}} t \sim 70$ for case $\mathrm{C}_{2 \mathrm{a}}$, and then saturates at an amplitude smaller than the maximum amplitude. The simple estimate $\Delta \epsilon \sim \sqrt{2 \hat{I}_{b} /\left(I_{A} \bar{\gamma}_{0}\right)}$ yields for the decoherence time from a differential energy change $\overline{\omega_{\beta, 0}} t_{\mathrm{d}, \epsilon} \sim 50$ [compare Eq. (17)], in good qualitative agreement with the simulation results.

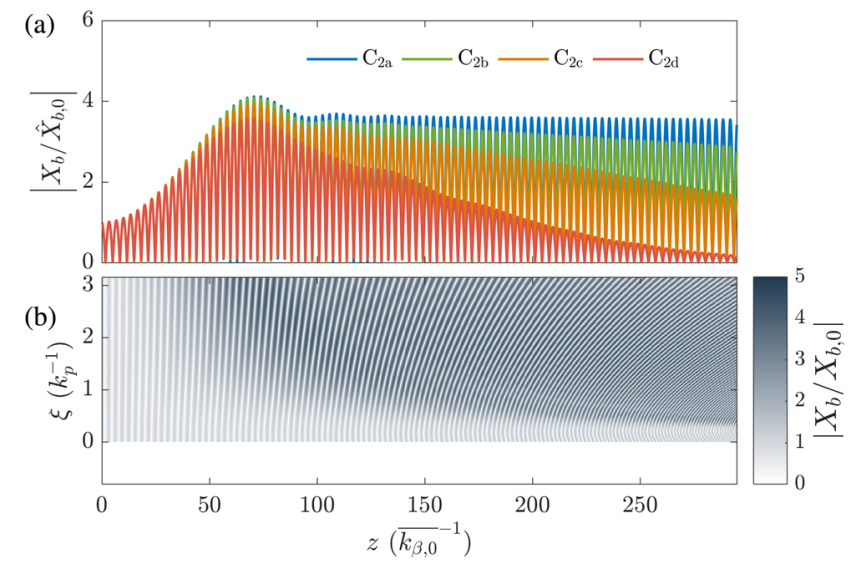

FIG. 5. (a) Evolution of beam centroid at the beam-tails (at $k_{p} \xi=4.0$ ) for cases $\mathrm{C}_{2 \mathrm{a}}$ (blue), $\mathrm{C}_{2 \mathrm{~b}}$ (green), $\mathrm{C}_{2 \mathrm{c}}$ (yellow), and $\mathrm{C}_{2 \mathrm{~d}}$ (red) obtained from PIC simulations. (b) Centroid evolution along $z$ and $\xi$ for case $\mathrm{C}_{2 \mathrm{a}}$, from a PIC simulation. 
It can also be seen that a subpercent relative energy spread $\left(0.5 \%\right.$ in case $\mathrm{C}_{2 \mathrm{~b}}$ ) already significantly reduces the beam centroid. An energy spread of $1.0 \%$ in case $\mathrm{C}_{2 \mathrm{c}}$ damps the centroid amplitude to about half the value near depletion as compared to case $\mathrm{C}_{2 \mathrm{a}}$. An energy spread of $2.0 \%$ in case $\mathrm{C}_{2 \mathrm{~d}}$ even reduces the centroid oscillation amplitude to a value lower than the initial beam centroid deviation.

In the limit of no energy change, the beam centroid is expected to be reduced by half an e-folding, compared to the case with no energy spread, at the decoherence time $\overline{\omega_{\beta, 0}} t_{\mathrm{d}, \Delta \gamma} \simeq 2 / \Delta \gamma$ [see Eq. (25)]. This yields $\bar{\omega}_{\beta, 0} t_{\mathrm{d}, \Delta \gamma} \simeq 400$ for case $\mathrm{C}_{2 \mathrm{~b}},{\overline{\omega_{\beta, 0}}}_{\mathrm{d}, \Delta \gamma} \simeq 200$ for case $\mathrm{C}_{2 \mathrm{c}}$ and $\overline{\omega_{\beta, 0}} t_{\mathrm{d}, \Delta \gamma} \simeq$ 100 for case $\mathrm{C}_{2 \mathrm{~d}}$. These numbers are in good agreement with the result shown in Fig. 5(a) (for case $\mathrm{C}_{2 b}$ the decoherence time is not reached).

The beam centroid evolution along the propagation distance and along the beam is depicted in Fig. 5(b) for case $\mathrm{C}_{2 \mathrm{a}}$. A similar behavior as in Fig. 3(b) is observed. Initially, $X_{b}$ grows for increasing $\xi$ and $z$, then $X_{b}(\xi, z)$ has a maximum at a propagation distance $z$ which depends on the comoving position $\xi$ along the beam. Hence, in contrast to the current conception, $X_{b}(\xi, z)$ does not grow steadily for greater propagation distances and positions along the beam. The propagation distance for which the growth of the hose instability is suppressed is greatest near to the maximum of $E_{z}$, as predicted by the two-particle model.

In summary, the simulation results presented in this section show that the presented hosing mitigation mechanisms are effective for a triangular beam-current profile and a beam energy of $28.5 \mathrm{GeV}$. Instead of an exponential amplification, as predicted by previous models, we observe a saturation of the hose instability. Moreover can a subpercent energy spread already significantly contribute to the mitigation of hosing and a percent-level energy spread can even reduce the beam centroid amplitude to a value smaller than the initial hosing seed. The observed hosing mitigation effects are in good agreement with the presented two-particle model.

Effective damping of the hose instability can only occur as long as the hosing seed is sufficiently small not to lead to beam breakup before the mitigation takes place. Reducing the initial hose seed is therefore crucial to fully stabilize the beam propagation. For this purpose we propose a concept in the following which employs plasma density tapers to mitigate initial drive-beam centroid offsets or misalignments between driver and witness beam that seed hosing.

\section{REDUCTION OF CENTROID OFFSETS AND MISALIGNMENTS WITH TAPERED PLASMA-DENSITY PROFILES}

\section{A. Theoretical model}

We consider a longitudinal plasma profile with a transition from the vacuum-plasma interface at $z_{v}$ to the flat-top region of the plasma at $z_{0}$. The beam centroid during the propagation in this vacuum-to-plasma transition can be described by

$$
\frac{d^{2} X_{b}}{d z^{2}}+k_{\beta}(z)^{2} X_{b}=0
$$

when neglecting the channel centroid displacement, the beam-energy change and effects from energy spread, where $k_{\beta}=\omega_{\beta} / c$ is the betatron wave number which depends on the $z$-coordinate through the dependency on the local plasma density $k_{\beta}^{2} / k_{\beta, 0}^{2}=n / n_{0}$, where $k_{\beta, 0}=k_{\beta}\left(z_{0}\right)$ and $n\left(z_{0}\right)=n_{0}$. Equation (26) corresponds to the system of a nonconservative harmonic oscillator with $z$-dependent frequency. The corresponding normalized Hamiltonian is given by

$$
\mathcal{H}(z)=\frac{X_{b}^{\prime}(z)^{2}}{2}+\frac{k_{\beta}(z)^{2} X_{b}(z)^{2}}{2},
$$

where $X_{b}^{\prime}=d X_{b} / d z$. Hence, the energy of the oscillator at the vacuum-plasma interface is given by $\mathcal{H}_{v}=X_{b, v}^{\prime 2} / 2+$ $k_{\beta, v}^{2} X_{b, v}^{2} / 2$, where $X_{b, v}=X_{b}\left(z_{v}\right)$ and $X_{b, v}^{\prime}=X_{b}^{\prime}\left(z_{v}\right)$ are the initial beam centroid and change of centroid, respectively, and where $k_{\beta, v}=k_{\beta}\left(z_{v}\right)$. By combining Eqs. (26) and (27) one finds that the energy changes according to $d \mathcal{H} / d z=$ $k_{\beta} k_{\beta}^{\prime} X_{b}^{2}$.

In short transitions with $L=z_{0}-z_{v} \ll k_{\beta, 0}^{-1}$ and $k_{\beta, v} \leq$ $k_{\beta, 0}, X_{b}$ and $X_{b}^{\prime}$ do not significantly evolve, and the energy of the oscillator at the beginning of the flat-top plasma profile at $z_{0}$ is essentially given by $\mathcal{H}_{\text {short }, 0}=X_{b, v}^{\prime 2} / 2+$ $k_{\beta, 0}^{2} X_{b, v}^{2} / 2$. However, for longer transitions with $k_{\beta, 0} L \gg 1$, the oscillator energy at the beginning of the flat-top profile $\mathcal{H}\left(z_{0}\right)$ can significantly differ from $\mathcal{H}_{\text {short }, 0}$. This is important for the mitigation of hosing because the hosing seed is proportional to the square root of the oscillator energy.

We consider the case for which the spatial hosing seed dominates over the velocity hosing seed, i.e., $X_{b, v}^{\prime 2} \ll$ $k_{\beta, 0}^{2} X_{b, v}^{2}$, such that $\mathcal{H}_{\text {short }, 0} \simeq k_{\beta, 0}^{2} X_{b, v}^{2} / 2$. In this case, $\mathcal{H}_{v} \leq$ $\mathcal{H}_{\text {short }, 0}$ if $k_{\beta, v} \leq k_{\beta, 0}$. Furthermore, if the vacuum-to-plasma taper is long $k_{\beta, 0} L \gg 1$ with a small relative local change $k_{\beta}^{-2} k_{\beta}^{\prime} \ll 1$ for all $z \in\left[z_{v}, z_{0}\right]$, the evolution of $X_{b}$ and $X_{b}^{\prime}$ occurs almost adiabatically. Hence, if $k_{\beta, v} \ll k_{\beta, 0}$ in such a scenario, the oscillator energy and therefore the hosing seed can significantly be reduced if a long taper is used compared to the case if no taper is used, i.e., $\mathcal{H}_{\text {long, } 0} \ll \mathcal{H}_{\text {short }, 0}$.

\section{B. Tapered profile}

As an example, we consider the functional dependence

$$
k_{\beta}(z)= \begin{cases}0 & \text { if } z \leq z_{v}, \\ k_{\beta, 0}\left[1-\left(z-z_{0}\right) / \lambda\right]^{-2} & \text { if } z_{v}<z \leq z_{0}, \\ k_{\beta, 0} & \text { if } z>z_{0},\end{cases}
$$


where $\lambda$ is the characteristic scale length of the taper (compare Refs. [32,33], in which this functional dependence was used for the matching of the beam betatron function). Such density profiles $\left(n=n_{0} k_{\beta}^{2} / k_{\beta, 0}^{2}\right)$ can be experimentally realized in appropriate gas capillaries $[34,35]$. Using the assumption that the initial centroid potential energy dominates over the initial centroid kinetic energy, the solution for this differential equation is (compare, e.g., [32])

$$
X_{b}(z)=X_{b, v}\left(1-\frac{z-z_{0}}{\lambda}\right)\left(\frac{\lambda \cos (\varphi)}{L+\lambda}+\frac{\sin (\varphi)}{k_{\beta, 0} \lambda}\right),
$$

with the phase advance $\varphi(z)=\int_{z_{v}}^{z} k_{\beta}\left(z^{\prime}\right) \mathrm{d} z^{\prime}$. In order to determine the optimal taper scale length $\lambda$ which minimizes the initial hose seed, we minimize the hosing seed at $z_{0}$ obtained with a tapered profile relative to the hosing seed obtained with a pure flat-top profile

$$
\eta_{0}=\sqrt{\frac{\mathcal{H}\left(z_{0}\right)}{\mathcal{H}_{\text {short }, 0}}}=\frac{\sqrt{k_{\beta, 0}^{2} X_{b}\left(z_{0}\right)^{2}+X_{b}^{\prime}\left(z_{0}\right)^{2}}}{k_{\beta, 0} X_{b, v}},
$$

for a given $X_{b, v}$ and for varying $\lambda$. This minimization yields the optimum parameter $\lambda_{\text {opt }} \simeq L / \sqrt{k_{\beta, 0} L}$ for long taper lengths compared to a betatron length, $k_{\beta, 0} L \gg 1$. When presuming this optimized taper parameter, the asymptotic expression for $\eta_{0}$ in the limit $k_{\beta, 0} L \gg 1$ is given by $\eta_{0, \text { asympt }} \simeq \sqrt{2} /\left(1+\sqrt{k_{\beta, 0} L}\right)$. The hosing seed reduction $\eta_{0}$ and the asymptotic solution $\eta_{0 \text {,asympt }}$ are depicted in Fig. 6(a) for varying taper lengths $k_{\beta, 0} L$. The graph predicts a reduction of the hosing seed to $\eta_{0} \sim 0.5$ for a taper length on the order of $k_{\beta, 0} L \sim 10$ and $\eta_{0} \sim 0.2$ for a taper length on the order of $k_{\beta, 0} L \sim 100$.

It should be noted that $k_{\beta, 0}^{-1}$ scales with the square root of the beam energy, and that tapers $k_{\beta, 0} L \gg 1$ are feasible even for energies in the TeV-range. The scaling of the taper length with the square root of the beam energy also has the beneficial implication that relative beam energy modulations within the taper can be neglected for great beam energies, as shown in the following. Considering that for the chosen density profile with $\lambda=\lambda_{\text {opt }}$ the relative energy change scales as $\left(\gamma_{0}-\gamma_{v}\right) / \gamma_{0} \sim \sqrt{2 / \gamma_{0}} k_{\beta, 0} L /$ $\left(1+\sqrt{k_{\beta, 0} L}\right)$, the energy modulation can be neglected for long tapers, if $\sqrt{k_{\beta, 0} L / \gamma_{0}} \ll 1$. Hence, for taper lengths $k_{\beta, 0} L \sim 100$, and highly relativistic beams, $\gamma_{0} \gg 1000$, the relative energy modulation within the tapered vacuum-toplasma transition and associated effects are insignificant.

\section{Comparison to PIC simulations}

This analytical prediction is benchmarked against results from PIC simulations using OSIRIS [21]. We consider a
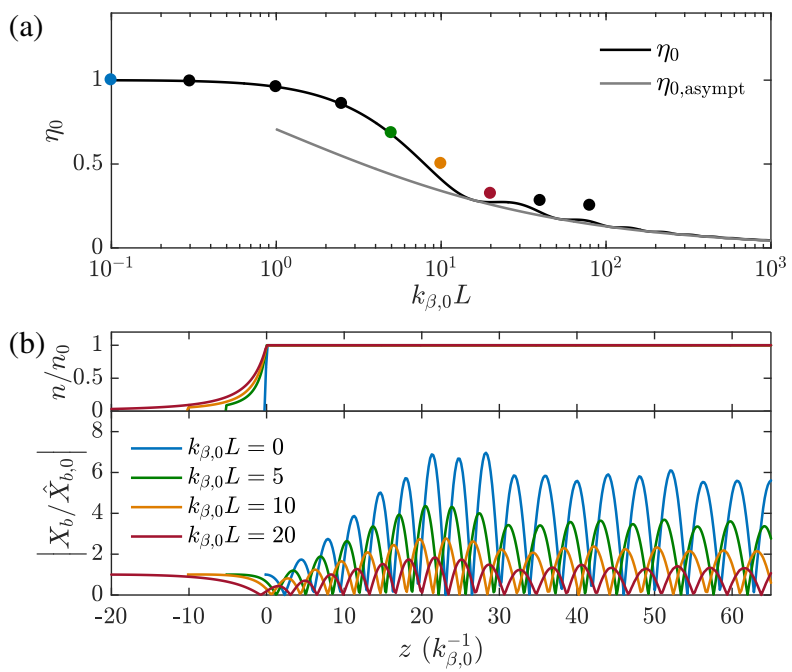

FIG. 6. Reduction of the hosing seeds in plasma density tapers with $\lambda=\lambda_{\text {opt }}$ at the tail of the beam $k_{p} \xi=4.0$. (a) Relative hosing seed $\eta_{0}$ according to Eq. (29) (black), respective asymptotic solution $\eta_{0 \text {,asympt }}$ (gray) and results from PIC simulations (circles). (b) Density profiles for different taper lengths (top) and respective beam centroid amplitudes from PIC simulations (bottom) for $k_{\beta, 0} L=0$ (blue), $k_{\beta, 0} L=5$ (green), $k_{\beta, 0} L=10$ (orange), and $k_{\beta, 0} L=20$ (red).

Gaussian electron beam with $\bar{\gamma}_{0}=1956.95$, no initial energy spread, a peak current of $I_{b}=0.25 \times I_{A}$, transverse dimensions of $k_{p} \sigma_{x}=k_{p} \sigma_{y}=0.1$, and longitudinal dimension of $k_{p} \sigma_{z}=1.0$ traversing a plasma target with a density profile that corresponds through $n=n_{0} k_{\beta}^{2} / k_{\beta, 0}^{2}$ to the above defined profile $k_{\beta}(z)$ [see Fig. 6(b), top]. The initial centroid along the beam is given by $k_{p} X_{b, 0}(\xi)=$ $0.01 \times \Theta(\xi)$. The centroid offset is introduced from the peak current location at $\xi=0$. The dimensions of the simulation box are $9 \times 9 \times 9 k_{p}^{-3}$ and the number of cells $512 \times 320 \times 320$. The chosen time step is $\omega_{p} \Delta t=0.0169$ using a numerical Cherenkov-radiation suppressing field solver [36]. The plasma is modeled with 4 particles per cell and the beam with 18 particles per cell using quadratic charge interpolation.

The PIC results in Fig. 6(a) are in good agreement with the analytical prediction for $\eta_{0}$ for $k_{\beta, 0} L \lesssim 1$. For $k_{\beta, 0} L \gg 1$, the PIC results and analytical model deviate owing to the occurrence of hosing in the tapered profile. Corresponding centroids, obtained from PIC simulations are depicted in Fig. 6(b), illustrating the substantial mitigation of the hose instability as a result of the plasma density tapering. The amplitude of the centroid oscillations is considerably reduced if density tapers with lengths of $k_{\beta, 0} L \gtrsim 1$ are employed, compared to the case with no taper.

We would like to note that the above investigated mitigation of centroid offsets in tapered vacuum-toplasma transitions analogously applies to the reduction 
of alignment offsets of witness beams with respect to the propagation axis of drive beams.

\section{MITIGATION OF WITNESS BEAM HOSING WITH ENERGY CHIRPS}

\section{A. Hosing of witness beams}

For the sake of energy conversion efficiency and for the reduction of accumulated correlated energy spread [37], witness beams with a sufficient current are required in PWFAs to (optimally) load the wake. According to Eq. (1), a witness-beam current, sufficiently high to cause beam loading inherently implies a strong coupling of the witness beam centroid to the plasma centroid (also confer Refs. $[14,22])$, and hence, implies that the witness beam itself drives hosing. The associated challenge of stable acceleration in the high-efficiency (beam-loaded) regime of plasma-based accelerators was also addressed in [38].

In contrast to the linear regime, in which the variation of the transverse wakefield along the witness beam enables a mitigation of hosing $[17,18]$, the transverse wakefield is constant along the beam in the blowout regime. As discussed in Sec. IV, an initial correlated energy spread can also mitigate hosing. We here investigate which degree of witness-beam energy chirp is required to mitigate hosing, and to allow for an emittance preservation to a few percent-level in the highly efficient beam-loaded regime.

\section{B. Head-to-tail decoherence}

We consider a witness beam with a flattop current profile which loads the plasma wake with high beam-loading efficiency [37], modifying the wakefield such that it varies approximately linearly along its axis (see Fig. 7). The witness beam therefore accumulates a linear chirp during the acceleration process which remains proportional to the mean beam energy.

We analyze the head-to-tail decoherence length in such a setup by means of the relations derived in Sec. IV. The head-to-tail variation of the longitudinal field along the witness beam $\Delta E_{w}$ implies a constant chirp according to $\chi_{w}=\left(k_{p} \gamma_{w}\right)^{-1} \partial \bar{\gamma} / \partial \xi=-\Delta E_{w} /\left(\bar{E}_{w} k_{p} l_{w}\right)$, where $\bar{E}_{w}$ is the mean accelerating field along the beam, $\gamma_{w}$ is the mean energy of the witness beam and $l_{w}$ is the length of the witness beam (compare Fig. 7). We use this expression for the chirp together with Eq. (23) to obtain the head-to-tail decoherence length of a beam with no uncorrelated energy spread

$$
k_{\beta, w} z_{\mathrm{d}, \chi} \simeq \frac{2 \pi}{\left|\chi_{w}\right| k_{p} l_{w}} .
$$

Considering a beam with a length of $k_{p} l_{w}=2$, this implies that a full head-to-tail decoherence is obtained after a distance $k_{\beta, w} z_{\mathrm{d}, \chi}^{a} \sim 157, k_{\beta, w} z_{\mathrm{d}, \chi}^{b} \sim 79$, and $k_{\beta, w} z_{\mathrm{d}, \chi}^{c} \sim 39$ for

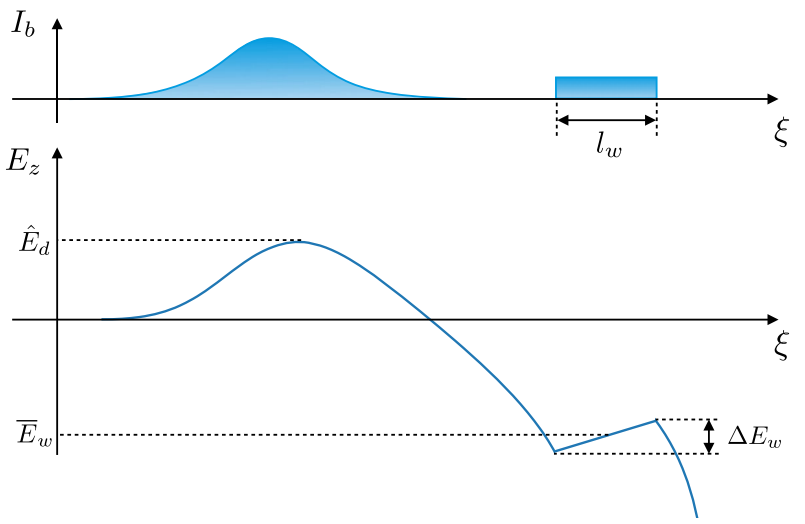

FIG. 7. Illustration of the current profiles of a drive and a witness beam pair (top) and resulting longitudinal wakefield structure in a PWFA (bottom). The peak decelerating field within the drive beam region is denoted by $\hat{E}_{d}$ and the average accelerating field within the witness beam by $\bar{E}_{w}$. The witness beam with flattop current profile and length $l_{w}$ loads the wake in such a way that the longitudinal field varies by $\Delta E_{w}$ from headto-tail.

relative chirps of $\chi_{w}^{a}=0.02, \chi_{w}^{b}=0.04$, and $\chi_{w}^{c}=0.08$, respectively. It should be noted that the head-to-tail decoherence length is not necessarily an accurate measure for the hosing mitigation length of a continuous beam [30], which rigorously also depends on the structure of the blowout wake. We therefore compare this scaling for the head-to-tail decoherence length results from 3D PIC simulations.

\section{Comparison to PIC simulations}

We compare the above estimates to 3D PIC simulations with the quasistatic code HIPACE [20]. The considered configuration is as follows. The drive beam is Gaussian with dimensions $k_{p} \sigma_{x, d}=k_{p} \sigma_{y, d}=0.8, k_{p} \sigma_{z, d}=\sqrt{2}$, with the center of the current profile at $k_{p} \xi=-5$ and with a peak density of $n_{d b} / n_{0}=4$. The witness beam has a trapezoidal current profile with a current of $1.6 \times I_{A}$ at the head, located at $k_{p} \xi=0$, and $1 \times I_{A}$ at the tail and has a length of $k_{p} l_{w}=2$. This current profile flattens the plasma wake and, regarding its impact on the hose instability, is equivalent to current profiles with a small slope of $E_{z}$ along the beam, i.e., for $\left|\chi_{w}\right|=\Delta E_{w} /\left(\bar{E}_{w} k_{p} l_{w}\right) \ll 1$. The beam has an initial transverse rms size of $k_{p} \sigma_{x, 0}=k_{p} \sigma_{y, 0}=0.01$, is matched to the wakefield, and has no uncorrelated energy spread. We investigate how the hosing of the beam with an initial offset of $X_{b, 0}=\sigma_{x, 0}$ with respect to the propagation axis of the drive beam evolves for differing constant chirps $\chi_{w}$. To guarantee the constancy of the chirp during the propagation in the plasma, and to isolate the effect of the chirp onto hosing, we disabled the energy gain of the beam in the simulations. The beam energy corresponds to $\gamma_{w}=$ 49000 (i.e., $25 \mathrm{GeV}$ ), but the results presented here are 
independent of the beam energy (as long as highly relativistic).

The numerical parameters of the simulations are as follows: the computational simulation box has dimensions of $16 k_{p}^{-1} \times 16 k_{p}^{-1} \times 11.5 k_{p}^{-1}$ with $512 \times 512 \times 600$ grid points. The witness beam consists of $10^{7}$ macroparticles and the plasma is modeled with 4 particles per cell within the interaction region and 1 particle per cell close to the computational box boundaries.

We study the beam dynamics for relative chirps $\chi_{w}^{0}=0$ (no chirp), $\chi_{w}^{a}=-0.02, \chi_{w}^{b}=-0.04$, and $\chi_{w}^{c}=-0.08$, i.e., with the slice energy decreasing from head to tail, as suggested by Fig. 7. The evolution of the beam centroids at the tail of the beams and the projected emittance are depicted in Fig. 8 top and bottom, respectively. For the beam without chirp, we observe an exponential growth of the centroid deviation at the tail. As seen in Fig. 8 (top), the introduction of an energy chirp leads to a reduction of the centroid growth rate and eventually to a disruption of hosing, i.e., to a saturation of the beam centroid offsets. For chirps $\chi_{w}^{b}=-0.04$ and $\chi_{w}^{c}=-0.08$ the head-to-tail decoherence owing to the chirp leads to a saturation of the centroid oscillations after the distances $k_{\beta, w} z^{b} \approx 110$ and $k_{\beta, w} z^{c} \approx 45$, respectively. This is compatible with the above head-to-tail decoherence estimates. For a relative chirp of $\chi_{w}^{a}=-0.02$ the growth is reduced. However, the beam centroid does not saturate at the head-to-tail decoherence length $k_{\beta, w} z_{\mathrm{d}, \chi}^{a} \sim 160$. This is owing to the
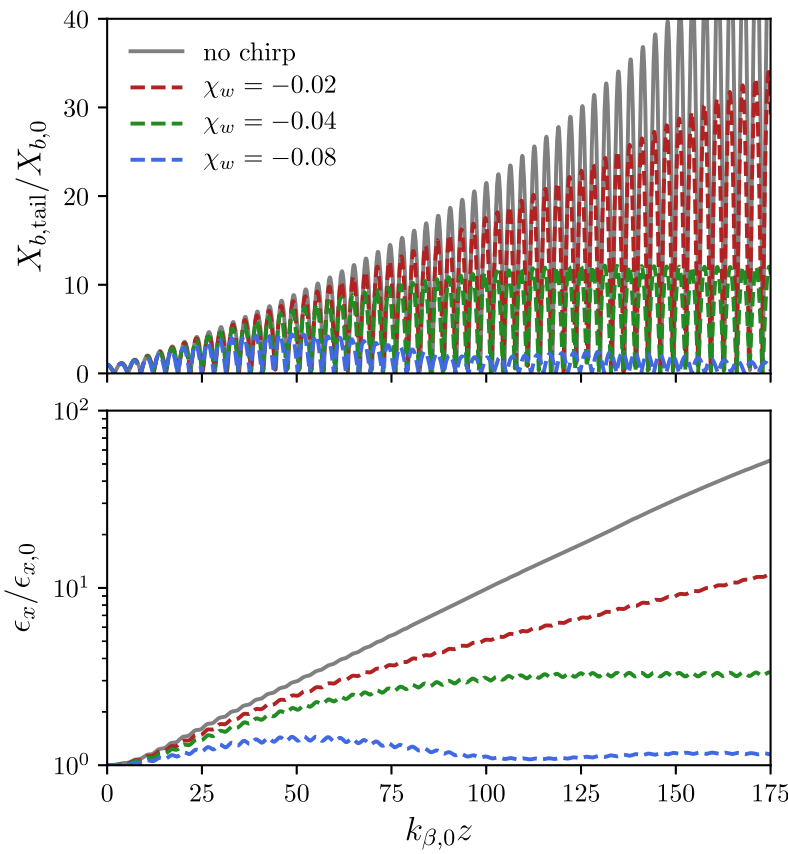

FIG. 8. PIC simulation results for beams with differing values of the relative chirp. The beams are initially misaligned with respect to the propagation axis of the drive beam and are subject to hosing. Centroid deviations at the tail of beams (top) and respective projected rms emittance (bottom). fact that slices along the continuous beam are coherently driving the tail slice beyond the two-particle head-to-tail decoherence length.

As shown in Fig. 8 (bottom), the projected transverse rms emittance grows approximately exponentially for the case with no energy chirp as the various beam slices start to be more and more misaligned with respect to each other owing to hosing. However, for the case with an energy chirp of $\chi_{w}^{a}=-0.02$, the emittance growth rate is significantly reduced, but the emittance after 28 betatron cycles still grows by one order of magnitude. For $\chi_{w}^{b}=-0.04$, the emittance growth after 28 betatron cycles is still $\epsilon_{x}^{b}\left(z=175 k_{\beta, 0}^{-1}\right) / \epsilon_{x, 0} \approx 3.2$. With an energy chirp of $\chi_{w}^{c}=-0.08$, the emittance grows by $8 \%$, i.e., $\epsilon_{x}^{c}\left(z=175 k_{\beta, 0}^{-1}\right) / \epsilon_{x, 0} \approx 1.08$. It should be noted that, since the hosing gain length is proportional to the betatron wavelength $2 \pi k_{\beta}^{-1} \propto \sqrt{\gamma_{w}}$, and since the number of betatron oscillations per stage decreases with increasing energy, the effects of hosing become less severe for higher beam energies in a plasma-based collider design.

In conclusion, relative energy chirps on the $\sim 10 \%$ level are required to allow for a reduction of the emittance growth to a sub-10\% level per stage in the regarded setup with a witness beam optimally loading the plasma wave and being initially misaligned by one rms width. The scheme is feasible, if matching sections enable the transport of beams with a $10 \%$-level energy chirp without significant quality deterioration (e.g., by use of optics with reduced chromatic dependence $[39,40]$ or with apochromatic matching sections [41]). An additional requirement for the viability of this scheme is that the chirp can be removed to a sufficient degree, e.g., with a plasma-dechirper [42], such that the energy spread is comparable to (or smaller than) the energy spread introduced at the interaction point owing to beam-beam effects in typical collider designs. For the realization of stable and quality-preserving highefficiency plasma-based accelerators, additional methods for the mitigation of hosing, such as the reduction of the initial misalignment by means of the method presented in VI, and the exploitation of ion-motion induced head-totail decoherence [43].

\section{SUMMARY AND CONCLUSION}

In this work, we explore mechanisms which lead to a mitigation of the hose instability of the drive beams and witness beams in PWFAs. The mitigating mechanisms from the intrinsic beam energy change, from an initially applied energy chirp or from an uncorrelated energy spread are discussed qualitatively and quantitatively by using a two-particle model.

We demonstrate with 3D particle-in-cell simulations that these mitigation mechanisms are effective for varying beam energies, differing types of hosing seed, and changing beam current profiles. We also investigate the spatiotemporal 
evolution of the beam centroids under the influence of the above effects and report the phenomenon that the beam centroid deviation develops a maximum close to the maximum decelerating field during propagation, which is contrasting to expectations from previous models. This phenomenon, as well as the mitigation times observed in PIC simulations, is in agreement with our models.

In addition, we discuss a method for the reduction of the initial spatial hosing seed in tapered vacuum-to-plasma profiles. It is shown analytically, and in agreement with 3D PIC simulations, that experimentally realizable profiles allow for a significant reduction of the hosing seed. It should be noted that this method and the respective scaling is also applicable for the reduction of misalignments between drive and witness beams in external injection of plasma-based accelerators.

Furthermore, we investigate the energy chirp required to sufficiently mitigate hosing of a witness beam in the highefficiency (beam-loaded) regime. As shown by use of particle-in-cell simulations and the derived scalings, a chirp on the $\sim 10 \%$-level, is required to sufficiently mitigate hosing of a beam misaligned by one rms width in the highefficiency regime, indicating the requirement for apochromatic matching sections [39-41] and dechirping methods [42], and/or additional hosing mitigation mechanisms, such as ion-motion [43].

In conclusion, this work discusses and establishes mechanisms for the mitigation of hosing of the drive beam in detail, and investigates possibilities to mitigate the hosing of witness beams in the high-efficiency regime of PWFAs.

\section{ACKNOWLEDGMENTS}

We would like to thank C. B. Schroeder for helpful discussions. This work was supported by the Director, Office of Science, Office of High Energy Physics, of the U.S. Department of Energy under Contract No. DE-AC0205CH11231 and the German Academic Exchange Service (DAAD) with funds from the German Federal Ministry of Education and Research (BMBF) and the People Programme (Marie Curie Actions) of the European Union's Seventh Framework Programme (FP7/20072013) under REA Grant Agreement No. 605728 (P. R. I. M.E.-Postdoctoral Researchers International Mobility Experience). We acknowledge the grant of computing time by the Jülich Supercomputing Centre on JUQUEEN under Project No. HHH23 and the use of the DESY IT highperformance computing facilities. This work was supported by LaserLab Europe IV_Grant Agreement No. 654148 (H2020-INFRAIA-2014-2015) and EuPRAXIA-Grant Agreement No. 653782 (H2020-INFRADEV-1-2014-1). J. V. acknowledges the support of FCT (Portugal) Grant No. SFRH/IF/01635/2015. Finally, we acknowledge the financial support by the Humboldt Foundation and by the Helmholtz Association through the Virtual Institute VH-VI-503.
[1] V. I. Veksler, Proceedings of the CERN Symposium On High-Energy Accelerators And Pion Physics (1956), p. 80.

[2] P. Chen, J. M. Dawson, R. W. Huff, and T. Katsouleas, Acceleration of Electrons by the Interaction of a Bunched Electron Beam with a Plasma, Phys. Rev. Lett. 54, 693 (1985).

[3] I. Blumenfeld, C. E. Clayton, F.-J. Decker, M. J. Hogan, C. Huang, R. Ischebeck, R. Iverson, C. Joshi, T. Katsouleas, N. Kirby, W. Lu, K. A. Marsh, W. B. Mori, P. Muggli, E. Oz, R. H. Siemann, D. Walz, and M. Zhou, Energy doubling of $42 \mathrm{GeV}$ electrons in a metre-scale plasma wakefield accelerator, Nature (London) 445, 741 (2007).

[4] J. B. Rosenzweig, B. Breizman, T. Katsouleas, and J. J. Su, Acceleration and focusing of electrons in two-dimensional nonlinear plasma wake fields, Phys. Rev. A 44, R6189 (1991).

[5] M. Litos et al., High-efficiency acceleration of an electron beam in a plasma wakefield accelerator, Nature (London) 515, 92 (2014).

[6] H. Suk, N. Barov, J. B. Rosenzweig, and E. Esarey, Plasma Electron Trapping and Acceleration in a Plasma Wake Field Using a Density Transition, Phys. Rev. Lett. 86, 1011 (2001).

[7] E. Oz et al., Ionization-Induced Electron Trapping in Ultrarelativistic Plasma Wakes, Phys. Rev. Lett. 98, 084801 (2007).

[8] B. Hidding, G. Pretzler, J. B. Rosenzweig, T. Königstein, D. Schiller, and D. L. Bruhwiler, Ultracold Electron Bunch Generation via Plasma Photocathode Emission and Acceleration in a Beam-Driven Plasma Blowout, Phys. Rev. Lett. 108, 035001 (2012).

[9] A. Martinez de la Ossa, J. Grebenyuk, T. Mehrling, L. Schaper, and J. Osterhoff, High-Quality Electron Beams from Beam-Driven Plasma Accelerators by WakefieldInduced Ionization Injection, Phys. Rev. Lett. 111, 245003 (2013).

[10] D. H. Whittum, W. M. Sharp, S. S. Yu, M. Lampe, and G. Joyce, Electron-hose instability in the ion-focused regime, Phys. Rev. Lett. 67, 991 (1991).

[11] M. Lampe, G. Joyce, S. P. Slinker, and D. H. Whittum, Electron-hose instability of a relativistic electron beam in an ion-focusing channel, Phys. Fluids B 5, 1888 (1993).

[12] A. A. Geraci and D. H. Whittum, Transverse dynamics of a relativistic electron beam in an underdense plasma channel, Phys. Plasmas 7, 3431 (2000).

[13] S. Deng, C. D. Barnes, C. E. Clayton, C. O’Connell, F. J. Decker, R. A. Fonseca, C. Huang, M. J. Hogan, R. Iverson, D. K. Johnson, C. Joshi, T. Katsouleas, P. Krejcik, W. Lu, W. B. Mori, P. Muggli, E. Oz, F. Tsung, D. Walz, and M. Zhou, Hose Instability and Wake Generation by an Intense Electron Beam in a Self-Ionized Gas, Phys. Rev. Lett. 96, 045001 (2006).

[14] C. Huang, W. Lu, M. Zhou, C. E. Clayton, C. Joshi, W. B. Mori, P. Muggli, S. Deng, E. Oz, T. Katsouleas, M. J. Hogan, I. Blumenfeld, F. J. Decker, R. Ischebeck, R. H. Iverson, N. A. Kirby, and D. Walz, Hosing Instability in the Blow-Out Regime for Plasma-Wakefield Acceleration, Phys. Rev. Lett. 99, 255001 (2007).

[15] T. J. Mehrling, R. A. Fonseca, A. Martinez de la Ossa, and J. Vieira, Mitigation of the Hose Instability in 
Plasma-Wakefield Accelerators, Phys. Rev. Lett. 118, 174801 (2017).

[16] V. E. Balakin, A. V. Novokhatsky, and V. P. Smirnov, Proceedings, 12th International Conference on HighEnergy Accelerators, HEACC 1983: Fermilab, Batavia (1984) (1983), pp. 119-120.

[17] J. Vieira, W. B. Mori, and P. Muggli, Hosing Instability Suppression in Self-Modulated Plasma Wakefields, Phys. Rev. Lett. 112, 205001 (2014).

[18] R. Lehe, C. B. Schroeder, J.-L. Vay, E. Esarey, and W. P. Leemans, Saturation of the Hosing Instability in Quasilinear Plasma Accelerators, Phys. Rev. Lett. 119, 244801 (2017).

[19] A. Martinez de la Ossa, T. J. Mehrling, and J. Osterhoff, Intrinsic Stabilization of the Drive Beam in Plasma-Wakefield Accelerators, Phys. Rev. Lett. 121, 064803 (2018).

[20] T. Mehrling, C. Benedetti, C. B. Schroeder, and J. Osterhoff, HiPACE: a quasi-static particle-in-cell code, Plasma Phys. Controlled Fusion 56, 084012 (2014).

[21] R. A. Fonseca, L. O. Silva, F. S. Tsung, V. K. Decyk, W. Lu, C. Ren, W. B. Mori, S. Deng, S. Lee, T. Katsouleas, and J. C. Adam, in Computational Science-ICCS 2002, Lecture Notes in Computer Science, edited by P. Sloot, A. Hoekstra, C. Tan, and J. Dongarra (Springer, Berlin Heidelberg, 2002), Vol. 2331, pp. 342-351; R. A. Fonseca, S. F. Martins, L. O. Silva, T. J. W., F. S. Tsung, and M.W.B., One-to-one direct modeling of experiments and astrophysical scenarios: pushing the envelope on kinetic plasma simulations, Plasma Phys. Controlled Fusion 50, 124034 (2008); R. A. Fonseca, J. Vieira, F. Fiuza, A. Davidson, F. S. Tsung, W. B. Mori, and L. O. Silva, Exploiting multi-scale parallelism for large scale numerical modelling of laser wakefield accelerators, Plasma Phys. Controlled Fusion 55, 124011 (2013).

[22] T. J. Mehrling, C. Benedetti, C. B. Schroeder, A. Martinez de la Ossa, J. Osterhoff, E. Esarey, and W. P. Leemans, Accurate modeling of the hose instability in plasma wakefield accelerators, Phys. Plasmas 25, 056703 (2018).

[23] Y. Glinec, J. Faure, A. Lifschitz, J. M. Vieira, R. A. Fonseca, L. O. Silva, and V. Malka, Direct observation of betatron oscillations in a laser-plasma electron accelerator, Europhys. Lett. 81, 64001 (2008).

[24] J. Vieira, C.-K. Huang, W. B. Mori, and L. O. Silva, Polarized beam conditioning in plasma based acceleration, Phys. Rev. ST Accel. Beams 14, 071303 (2011).

[25] P. Michel, C. B. Schroeder, B. A. Shadwick, E. Esarey, and W. P. Leemans, Radiative damping and electron beam dynamics in plasma-based accelerators, Phys. Rev. E 74, 026501 (2006).

[26] K. V. Lotov, Blowout regimes of plasma wakefield acceleration, Phys. Rev. E 69, 046405 (2004).

[27] A. Martinez de la Ossa, T. J. Mehrling, L. Schaper, M. J. V. Streeter, and J. Osterhoff, Wakefield-induced ionization injection in beam-driven plasma accelerators, Phys. Plasmas 22, 093107 (2015).

[28] M. J. Hogan, T. O. Raubenheimer, A. Seryi, P. Muggli, T. Katsouleas, C. Huang, W. Lu, W. An, K. A. Marsh, W. B. Mori, C. E. Clayton, and C. Joshi, Plasma wakefield acceleration experiments at FACET, New J. Phys. 12, 055030 (2010).
[29] E. Adli, J.-P. Delahaye, S. J. Gessner, M. J. Hogan, T. Raubenheimer, W. An, C. Joshi, and W. Mori, in Proceedings, Community Summer Study 2013: Snowmass on the Mississippi (CSS2013): Minneapolis, MN, USA, 2013, https://inspirehep.net/record/1246947.

[30] A. Chao and M. Tigner, Handbook of Accelerator Physics and Engineering, Handbook of Accelerator Physics and Engineering (World Scientific, Singapore, 1999).

[31] SLAC, Technical Design Report for the FACET-II Project at SLAC National Accelerator Laboratory, 2016.

[32] K. Floettmann, Adiabatic matching section for plasma accelerated beams, Phys. Rev. ST Accel. Beams 17, 054402 (2014).

[33] X. L. Xu, J. F. Hua, Y. P. Wu, C. J. Zhang, F. Li, Y. Wan, C.-H. Pai, W. Lu, W. An, P. Yu, M. J. Hogan, C. Joshi, and W. B. Mori, Physics of Phase Space Matching for Staging Plasma and Traditional Accelerator Components Using Longitudinally Tailored Plasma Profiles, Phys. Rev. Lett. 116, 124801 (2016).

[34] P.-F. Hao, Y.-T. Ding, Z.-H. Yao, F. He, and K.-Q. Zhu, Size effect on gas flow in micro nozzles, J. Micromech. Microeng. 15, 2069 (2005).

[35] L. Schaper, L. Goldberg, T. Kleinwchter, J.-P. Schwinkendorf, and J. Osterhoff, Longitudinal gas-density profilometry for plasma-wakefield acceleration targets, Nucl. Instrum. Methods Phys. Res., Sect. A 740, 208 (2014).

[36] R. Lehe, A. Lifschitz, C. Thaury, V. Malka, and X. Davoine, Numerical growth of emittance in simulations of laser-wakefield acceleration, Phys. Rev. ST Accel. Beams 16, 021301 (2013).

[37] M. Tzoufras, W. Lu, F. S. Tsung, C. Huang, W. B. Mori, T. Katsouleas, J. Vieira, R. A. Fonseca, and L. O. Silva, Beam Loading in the Nonlinear Regime of Plasma-Based Acceleration, Phys. Rev. Lett. 101, 145002 (2008).

[38] V. Lebedev, A. Burov, and S. Nagaitsev, Efficiency versus instability in plasma accelerators, Phys. Rev. Accel. Beams 20, 121301 (2017).

[39] J. van Tilborg, S. Steinke, C. G. R. Geddes, N. H. Matlis, B. H. Shaw, A. J. Gonsalves, J. V. Huijts, K. Nakamura, J. Daniels, C. B. Schroeder, C. Benedetti, E. Esarey, S. S. Bulanov, N. A. Bobrova, P. V. Sasorov, and W. P. Leemans, Active Plasma Lensing for Relativistic Laser-PlasmaAccelerated Electron Beams, Phys. Rev. Lett. 115, 184802 (2015).

[40] C. A. Lindstrøm, E. Adli, G. Boyle, R. Corsini, A. E. Dyson, W. Farabolini, S. M. Hooker, M. Meisel, J. Osterhoff, J.-H. Röckemann, L. Schaper, and K. N. Sjobak, Emittance Preservation in an Aberration-Free Active Plasma Lens, Phys. Rev. Lett. 121, 194801 (2018).

[41] C. A. Lindstrøm and E. Adli, Design of general apochromatic drift-quadrupole beam lines, Phys. Rev. Accel. Beams 19, 071002 (2016).

[42] R. D'Arcy et al., Tunable Plasma-Based Energy Dechirper, Phys. Rev. Lett. 122, 034801 (2019).

[43] T. J. Mehrling, C. Benedetti, C. B. Schroeder, E. Esarey, and W.P. Leemans, Suppression of Beam Hosing in Plasma Accelerators with Ion Motion, Phys. Rev. Lett. 121, 264802 (2018). 